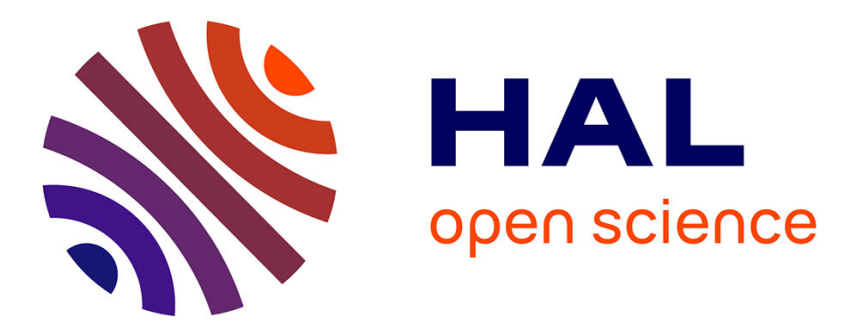

\title{
Ex-voto en bois de Chamalières (Puy-de-Dôme) et des Sources de la Seine (Côte-d'Or) : essai de comparaison
} Anne-Marie Romeuf

\section{To cite this version:}

Anne-Marie Romeuf. Ex-voto en bois de Chamalières (Puy-de-Dôme) et des Sources de la Seine (Côted'Or) : essai de comparaison. Gallia - Fouilles et monuments archéologiques en France métropolitaine, 1986, 44 (1), pp.65-89. 10.3406/galia.1986.2853 . hal-01940892

\section{HAL Id: hal-01940892 \\ https://hal.science/hal-01940892}

Submitted on 27 Feb 2020

HAL is a multi-disciplinary open access archive for the deposit and dissemination of scientific research documents, whether they are published or not. The documents may come from teaching and research institutions in France or abroad, or from public or private research centers.
L'archive ouverte pluridisciplinaire HAL, est destinée au dépôt et à la diffusion de documents scientifiques de niveau recherche, publiés ou non, émanant des établissements d'enseignement et de recherche français ou étrangers, des laboratoires publics ou privés.

\section{(1) (1) $\$$}

Distributed under a Creative Commons Attribution - NonCommercial - NoDerivatives| 4.0 


\title{
EX-VOTO EN BOIS DE CHAMALIÈRES (Puy-de-Dôme) ET DES SOURCES DE LA SEINE. ESSAI DE COMPARAISON
}

\author{
par Anne-Marie ROMEUF
}

En 1963 on découvrait les premiers bois sculptés des Sources de la Seine (Côte-d'Or) lors d'un aménagement du site, et en 1968, à l'occasion de travaux de construction, les ex-voto de la Source des Roches, à Chamalières (Puy-de-Dôme). Les premiers ont été récemment publiés dans leur intégralité ${ }^{1}$; plusieurs comptes rendus de la découverte et un catalogue d'exposition, permettant d'apprécier leur exceptionnel intérêt, ont déjà été consacrés aux seconds's, mais la publication d'ensemble est retardée par le nombre considérable de sculptures et la fragilité du matériau qui rendent difficile toute manipulation ${ }^{3}$. Il nous a donc semblé intéressant d'annoncer cette publication, maintenant que nous avons une vision exhaustive de la découverte des Sources de la Seine, par une comparaison entre les bois sculptés provenant des deux sites, ces ex-voto qui, suivant des destins parallèles, ont été déposés et retrouvés presque simultanément dans les deux régions.

$$
* *
$$

Manifestation dans les deux cas d'un culte des eaux, ils avaient été offerts à la Source, les premiers dans le fond plat d'une vallée étroite où naît la Seine dont les eaux ne présentent aucune valeur thérapeutique, les autres dans la cuvette naturelle d'une source minérale jaillissant sur un terrain des bords de Limagne à l'extrémité de la coulée de lave d'un volcan, et dont les vertus de l'eau chlorobicarbonatée sodique, calcique et magnésienne sont reconnues. Les deux sites étaient sauvages et marécageux mais la Source des Roches à Chamalières était proche de la ville d'Augustonemetum, et cette proximité, jointe à la qualité de ses eaux, peut expliquer l'attrait particulier qu'elle exerça et la quantité d'ex-voto qui lui furent dédiés.

Des découvertes anciennes en Bourgogne au XviII ${ }^{\mathrm{e}}$ s., comme en Auvergne au XIXe s., avaient annoncé les récentes, mais aux Sources de la Seine il y eut fouille dès 1836 et découverte de nombreux ex-voto de pierre et de bronze, alors qu'à Chamalières on signalait seulement en 1843 la découverte fortuite d'ex-voto de bois, dont l'antiquité fut ensuite mise en doute.

1 Simone Deyts : Les bois sculptés des Sources de la Seine, XLI e supplément à Gallia, Paris, 1983.

2 C. VATin : Informations dans Gallia, 27, 1969, 312-335; Ex-voto de bois gallo-romains à Chamalières dans Revue Archéologique, 1969, p. 103-114; Wooden sculplure from gallo-roman Auvergne dans Antiquily, 1972, p. 39-42, photo en frontispice, et pl. II-IX. - J.-C. Poursat : Informalions, dans Gallia, 31, 1973, p. 439-444; Les ex-volo en bois galloromains de Chamalières (Puy-de-Dôme) dans La revue du
Louvre et des Musées de France, décembre 1979, no 516, p. 353-356. - Musée Bargoin. Ex-volo gallo-romains de la Source des Roches à Chamalières. Catalogue d'exposition par M. Dumontet et A.-M. Romeuf, préface de R. Martin, introduction de J.-C. Poursat, Clermont-Ferrand, 1980.

3 Ce travail en cours de réalisation est freiné par la nécessité de l'établissement d'un catalogue complet qui est effectué en collaboration avec M. Dumontet. 
Les fouilles récentes ont montré par l'étude des monnaies, de la céramique, et du petit matériel, que les deux dépôts d'ex-voto de bois étaient contemporains, non postérieurs au début de l'époque flavienne, même s'il existe de nombreux éléments plus précoces à Chamalières. Par une curieuse coïncidence, on constate même sur chacun des deux sites la présente de deux fibules "à queue de paon " de même type, en or à la Seine, en bronze à la Source-des Roches, qui sont en Bourgogne les seules fibules découvertes dans la fouille et qui constituent la moitié de celles trouvées en Auvergne.

Dans les deux cas également les bois étaient très groupés, tous localisés en un même point, mais plus amoncelés encore à Chamalières, dans la cuvette de la source qui était vraisemblablement leur lieu de dépôt originel, alors que les statues des Sources de la Seine retrouvées en terrain plat paraissent y avoir été apportées accidentellement par les alluvions de la source principale.

Différence plus importante, il existait aux Sources de la Seine plusieurs constructions semblant contemporaines des ex-voto de bois - un fanum et deux bassins - alors qu'à Chamalières où il n'y avait aucun bâtiment, le seul aménagement du sile était un cailloutis qui consolidait le bord de la mare que devait former la source, tout en délimitant l'espace sacré. Et si le culte est resté prospère aux Sources de la Seine jusqu'à la fin du II ${ }^{\mathrm{s}} \mathrm{s}$. avec l'édification de plusieurs bâtiments d'une certaine richesse, voire jusqu'au Ive s. s'il faut en croire les découvertes monétaires, il a presque disparu à Chamalières après l'enfouissement des ex-voto de bois puisqu'il n'est plus attesté ensuite que par quelques tessons de céramique et de rares monnaies.

Enfin le dépôt de Chamalières est très abondant avec ses dix mille sculptures ou fragments, mais il est beaucoup moins complet et diversifié que celui des Sources de la Seine qui comprenait, outre les bois sculptés, de nombreux ex-voto de pierre et de bronze. La rareté de la pierre tendre en Auvergne explique peut-être cette absence à Chamalières, mais la présence d'un seul ex-voto de bronze tendrait plutôt à prouver qu'il y avait une spécificité du matériau, et que l'on n'offrait que du bois à Chamalières, ou que les fidèles étaient plus pauvres puisqu'aux fibules de bronze de ce site correspondent des fibules en or aux Sources de la Seine. Cette particularité peut tenir aussi à des raisons chronologiques, étant donné que les ex-voto de pierre aux Sources de la Seine sont considérés comme plus tardifs ${ }^{5}$ - encore que deux sculptures de pierre aient été retrouvées mêlées aux ex voto de bois - et qu'il semble bien que la statuaire de bois ait été généralement abandonnée en Gaule à la fin du i ${ }^{\text {er }}$ s., selon un phénomène que l'on constate aussi à Luxeuil-les-Bains (Haute-Saône) et à Essarois (Côte-d'Or) et qui peut correspondrc à un changement de goût ou de croyance ${ }^{6}$.

Ainsi les ex-voto des Sources de la Seine et de la Source des Roches apparaissent-ils situés dans des contextes légèrement différents. Les premiers n'étaient qu'une partie d'un lot d'ex-voto sculptés dans de multiples matériaux et déposés dans un sanctuaire bâti qui a été fréquenté plusieurs siècles durant, dans un lieu isolé, par des fidèles plus riches. Les autres sont à eux seuls la manifestation d'un culte très important mais plus éphémère, à proximité d'une ville.

4 Selon M. A. Dollfuss (Catalogue des fibules de bronze de IIaule-Normandie. Paris, 1973 , p. 20; ces fibules à queue de paon auraient pu avoir un usage plus votif que vestimentaire, ce qui expliquerait en partie cette coincidence.

5 R. Martin (Introduction, p. 2, au catalogue Ex-voto du sanctuaire des Sources de la Seine, Musée Archéologique de Dijon, 1966) indique que les ex-voto de pierre ont été trouvés autour du bassin dont le sol était tapissé de monnaies de Constantin. - H. Baudot (Rapporl sur les découvertes archéologiques failes aux Sources de la Seine dans Mémoires de la Commission des Antiquités de la Côte d'Or, t. 2, 1842-46, p. 95-144 et pl. I à XVII; explique que les ex-voto de bronzc étaient presque tous groupés dans un vase contenant un trésor de monnaies datées des I $^{\text {er }}$ au IV $^{\text {e }}$ s. (p. 119-120 et pl. X).

6 Coincidant soit avec une influence romaine sur le style des sculptures, soit avec un événement politique ou religieux comme la persécution des druides, par exemple. La découverte récente d'ex-voto de bois du II $\mathrm{s}$. à Montlay-en-Auxois (Còte-d'Or) démontre cependant l'utilisation de ce matériau au-delà du I $^{\text {er }}$ s. - J. DupoNr, in sanctuaire de source dans Archeologia, 214, juin 1986, p. 55 à 59. - Informations dans Gallia, 43, 1985, p. 257-259, fig. 8 . 


\section{TYPOLOGIE DES EX-VOTO. NOMBRE ET PROPORTIONS.}

Le nombre des ex-voto est beaucoup plus important à Chamalières. On y compte environ 1550 sculptures et 8850 fragments, soit vraisemblablement les restes de 3000 ex-voto ${ }^{7}$ contre moins de 350 en bois aux Sources de la Seine : près de dix fois plus en Auvergne qu'en Bourgogne, et encore trois fois plus, tous matériaux confondus si l'on incluait dans le nombre des ex-voto des Sources de la Seine ceux de pierre et de bronze.

Les thèmes représentés dans la sculpture de bois sont les mêmes à quelques variantes près, mais les proportions sont différentes ainsi que le montre le tableau ci-après. Les deux sites ont livré des figurations de personnages entiers, de têtes, de troncs ou de bassins, de bras, de jambes, de planches anatomiques et d'animaux, ainsi que des plaquettes et quelques objets mobiliers. Mais les séries sont numériquement assez proches aux Sources de la Seine et beaucoup plus hétérogènes à Chamalières, où les écarts sont même très accusés. Sur ce dernier site, les bras et les jambes constituent les $2 / 3$ des ex-voto, les personnages $1 / 6$ et les têtes $1 / 10$, alors qu'aux Sources de la Seine les têtes sont les plus représentées (plus du quart) puis, presque à égalité, les personnages entiers, les planches anatomiques, et, les membres. Il y a beaucoup plus de têtes groupées aux Sources de la Seine qu'à Chamalières, nettement plus de planches anatomiques, et plus d'animaux.

Si l'on prend en compte les sculptures de pierre, on constate toujours des disproportions entre les séries des ex-voto des Sources de la Seine et celles de ceux de Chamalières, mais elles s'atténuent puisque le nombre de membres s'accroît considérablement aux Sources de la Seine, qu'il n'y a aucune planche anatomique supplémentaire, et très peu de têtes groupées et d'animaux.

Enfin, si l'on ne comparait que les ex-voto de pierre des Sources de la Seine à ceux de bois de Chamalières, les membres viendraient en tête dans les deux cas, mais en moindre proportion aux Sources de la Seine, et il y aurait aussi certaines catégories non représentées sur ce dernier site planches anatomiques et têtes superposées - qui apparaissent donc comme spécifiques de la sculpture de bois.

\section{COMPARAISON ENTRE LES EX-VOTO, SÉRIE PAR SÉRIE.}

Il existe d'abord des analogies typologiques entre des séries qui ne peuvent donner lieu à des comparaisons stylistiques. C'est notamment le cas des plaquettes minces et rectangulaires, vraisemblablement peintes ou inscrites à l'origine, et qui ont été recueillies en nombre non négligeable dans les deux cas : soixante à Chamalières, dont une avec des restes de peinture, "un grand nombre " aux Sources de la Seine ${ }^{8}$. Autre similitude, dans chacun des deux dépôts, l'une d'entre elles servait de support à des yeux de bronzeg.

Parmi les objets mobiliers qui ont été recensés, certains peuvent être comparés : pilier quadrangulaire avec mortaise à la Seine ${ }^{10}$ et planches et pièces d'assemblage avec tenons el mortuises à la Source des Roches, motif décoratif en S aussi dans les deux cas ${ }^{11}$. D'autres n'existent que sur l'un des deux sites : émigrette aux Sources de la Seine, récipients et maillets à Chamalières ${ }^{12}$.

7 On peut raisonnablement estimer en effet que les ex-voto se sont brisés en 5 ou 6 fragments en moyenne, ce qui permet d'arriver à cette évaluation.

8 S. Deyts : op. cil. p. 126.

9 Ibid, no 298, et Musée Bargoin no 188. Il s'agit aux Sources de la Seine d'une plaquette rectangulaire alors que celle de Chamalières est découpée en forme de cœur.

10 Ibid., op. cil., n*307, et Musée Bargoin nos 206 à 209 et 212 .

11 Ibid., op. cil. no 301 ; inédit à Chamalières.

12 Ibid., op. cit., no 299, et Musée Bargoin nos $198-199$ et 204-205. 
Enfin, mêlés aux ex-voto dans les deux sources, ont été recueillies de nombreuses branches. Le fait est vraisemblablement fortuit, mais il est possible aussi que quelques-unes aient été jetées comme offrandes, soit parce qu'elles avaient une forme de jambe par exemple, soit parce qu'étant de même matériau que les autres ex-voto, elles ont pu être considérées par le donateur comme pouvant en faire office. Aux Sources de la Seine, certaines branches pourraient être des débuts d'ébauches d'un atelier situé à proximité13, alors que cette interprétation paraît peu vraisemblable à Chamalières.

Parmi les ex-voto non anthropomorphes, la dernière catégoric est celle des animaux qui permet déjà un début de comparaison stylistique (fig. 1). Ils sont plus nombreux aux Sources de la Seine, deux fois plus en chiffre absolu, mais dans les deux cas on a affairc aux mêmes espèces animales, chevaux et bovidés. Les premiers sont représentés soit entiers, soit sous forme de protomé à la Seine ou de jambe de cheval à Chamalières, ou encore cheval monté par un cavalier sur ce dernier site ${ }^{14}$. Mais alors que les bovidés sont nombreux en Bourgogne, on ne connait qu'une patte d'animal de cette famille en Auvergne ${ }^{15}$. De surcroît, les chevaux des Sources de la Seine sont plus finis, modelés et réalistes, avec détail de la tête (chanfrein, yeux, bouche, joues, crinière, museau) et du corps (poitrail, flancs, jambes) ${ }^{16}$ alors que ceux de Chamalières restent trapus, disproportionnés, ct peu esthétiques, avec un corps massif et des jambes rectilignes. Cependant on peut rapprocher la silhouette stylisée de deux chevaux, l'un très abîmé provenant des Sources de la Scine (fig. 1, 1), et l'autre, le plus réussi du lot de Chamalières (fig. 1, 2), pour leur contour sinueux et leur petite têtc au museau pointu.
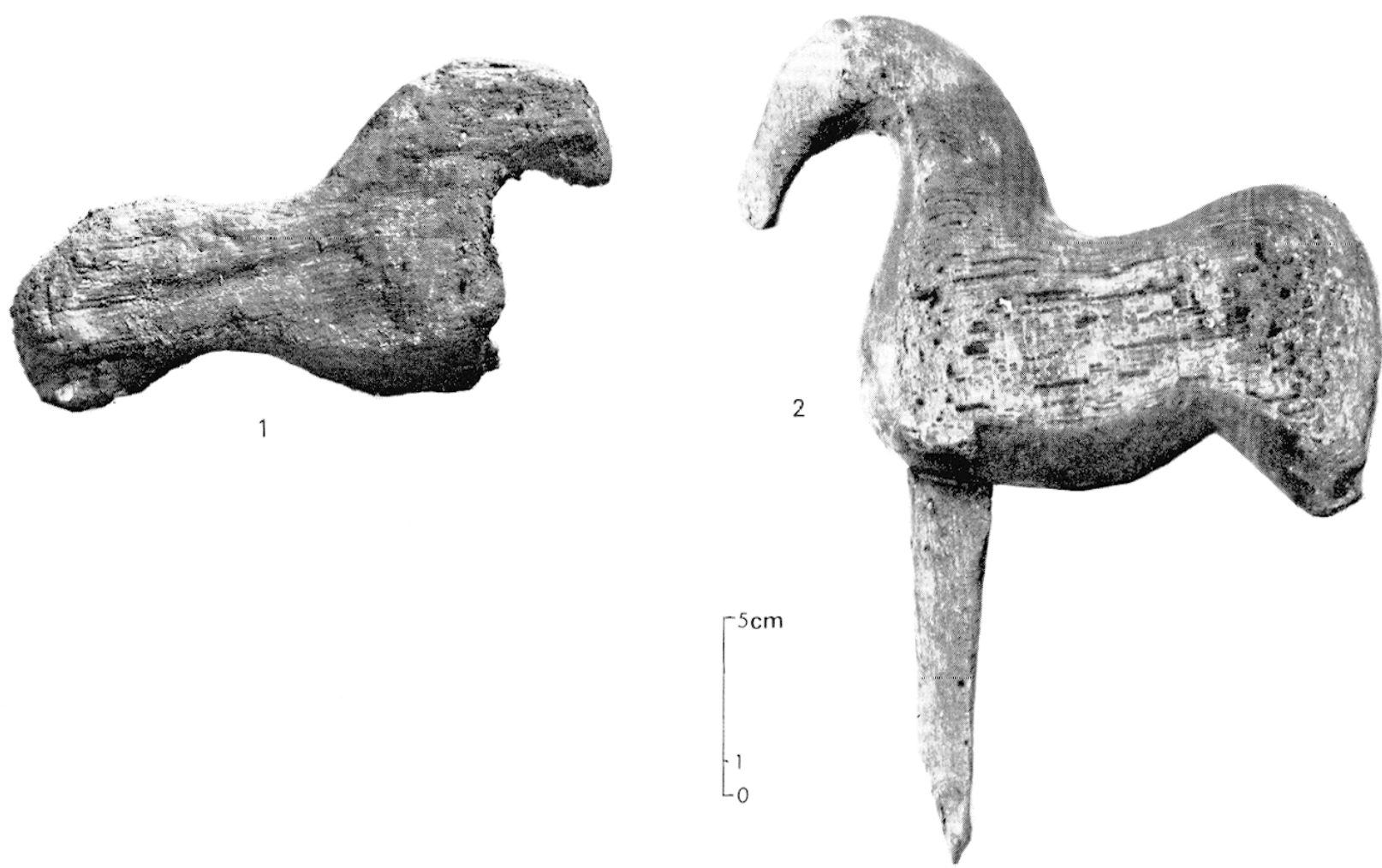

I Chevaux : 1, Sources de la Seinc. Haut. conservée : $0,09 \mathrm{~m} ; 2$, Chamalières. Haut. conservée : 0,26 m (échelle commune).

13 Ibid., op. cil., nº 308 et p. 150.

14 Musée Bargoin $\mathrm{n}^{\circ} 189$.
15 Inédite.

16 S. Dryrs, op. cit., nos 268 à 270 el 274. 
Mais ce sont encore les ex-voto anthropomorphes qui fournissent les comparaisons les plus intéressantes.

Les jambes (fign. 2) sont lia catćgoric de loin la plus abondante à Chamalières (fig. 2, 4, 6 et 8), et elles sont assez nombreuses aussi aux Sources de la Seine (fig. 2, 3, 5 et $\mathbf{y}$ ), mais moins cependant que d'autres séries d'ex-voto. Elles sont de même type sur les deux sites, représentées généralement du haut de la cuisse à l'extrémité du pied qui est souvent en extension, et il arrive que la hanche soit figurée. Pour quelques exemplaires isolés à Chamalières, la cuisse est coupée bas, juste au-dessus du genou, mais c'est une particularité que l'on peut considérer comme négligeable, compte tenu de sa rareté. De même une jambe provenant des Sources de la Seine qui repose sur un socle ${ }^{17}$ n'a pas d'équivalent à Chamalières mais pourrait être rapprochée des moitiés inférieures de corps terminées par un socle trouvées en Auvergne. Quant aux pieds représentés seuls, ils ne le sont qu'à de très rares exemplaires, moins de dix à Chamalières, un aux Sources de la Seine. Enfin, dernier point commun, aucune affection pathologique ne peut être signalée en l'un ou l'autre cas.

La technique et le style de ces sculptures présentent également bien des similitudes. Les deux dépôts ont en commun d'avoir livré à côté de très belles jambes proportionnées el modelées asséz réalistes mais peu nombreuses, des œuvres plus stylisées et moins bien proportionnées qui restent tributaires de la forme du matériau et qui constituent la majorité de la production, ainsi que quelques exemplaires très frustes sculptés dans des branches qui ont déterminé leur conformation. C'est ainsi que l'on peut établir entre les jambes trouvées sur les deux sites des parallèles intéressants. Certaines d'entre elles (fig. 2, 3 et 4) sont réalistes, avec la cuisse et le mollet galbés, le genou proéminent, et le pied formant un angle par rapport à la jambe ${ }^{18}$; deux jambes de cette série comprennent même la représentation de l'aine'19. I'autres ne font que suivre la ligne de la branche noueuse utilisée pour les fabriquer, et conservent des traces de coups d'outil sur des méplats laissés bruts ${ }^{20}$. Deux autres enfin ont été taillées dans des branches courbes à peine aplanies et dégrossies, terminées par un pied schématisé uniquement par la figuration de sa plante taillée en biseau (fig. $2, \mathbf{5}$ et $\mathbf{6}$ ), tandis que les deux dernières ont un pied gigantesque, pointu et sans aucun réalisme qui résulte à l'évidence de l'utilisation peu évoluée d'une branche fourchue (fig. 2, 7 et 8).

Cependant ces rapprochements, aussi nombreux et convaincants qu'ils soient, ne peuvent masquer une différence fondamentale : on retrouve à la Source des Roches à peu près tous les types connus aux Sources de la Seine, mais le style caractéristique de Chamalières, - celui des jambes sculptées dans des planches de hêtre à section triangulaire dont la ligne saillante sert à figurer l'os du tibia et le devant du pied en extension, tandis que la partie épaisse correspond au galbe de l'arrière de la cuisse et du mollet (fig. 9, 48 et 49) -, n'existe dans aucun exemplaire en Bourgogne.

Les bras présentent également des similitudes dans leur typologie bien qu'ils soient assez rares aux Sources de la Seine et très nombreux à Chamalières, avec des représentations de ce fait beaucoup plus diversifiées (fig. 3). Dans les deux cas, ils sont le plus généralement figurés depuis l'épaule jusqu'à l'extrémité des doigts, tendus, et sans autre détail. Dans les deux cas également il existe des mains traitées pour elles-mêmes, sans le bras, un exemplaire à la Seine, et quelques unités à Chamalières. Mais, alors que l'épaule est fréquemment indiquée sur ce dernier site (fig. $3, \mathbf{1 0}$ et 14; fig. 9, 50, 51 et 52), elle n'apparaît jamais aux Sources de la Seine. Souvent aussi à la Source des Roches le bras est figuré vêtu avec l'extrémité d'une manche tombant sur la main jusqu'à la naissance des doigts (fig. 3, 14; fig. 9, 51) et il est intéressant de noter une représentation de ce type, même isolée, aux Sources

17 Ibid., op. cil., no213.

18 Ibid., op. cit., no 212, ot. Informalions dans Gallia, 27, 1969, p. 328 .
19 Ibid., op. cit., no 225, et Musée Bargoin no 101.

20 Ibid., op. cit., no 213, et Revue du louvre, 1979, p. 354, fig. 3 b; ou ibid., op. cil., no 222, et Musée Bargoin no 110 . 


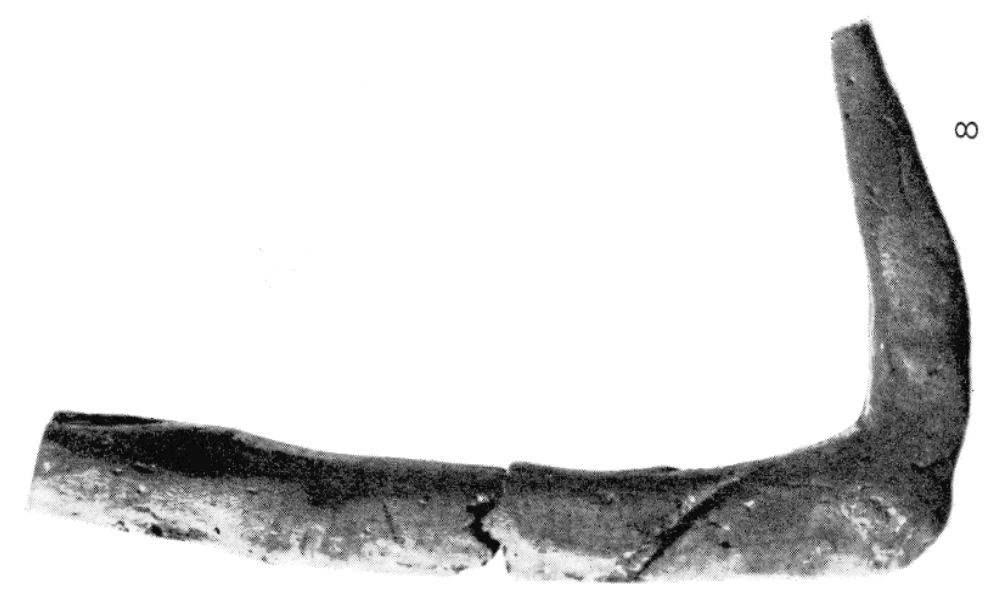

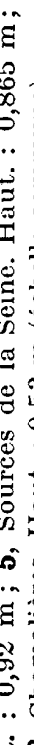
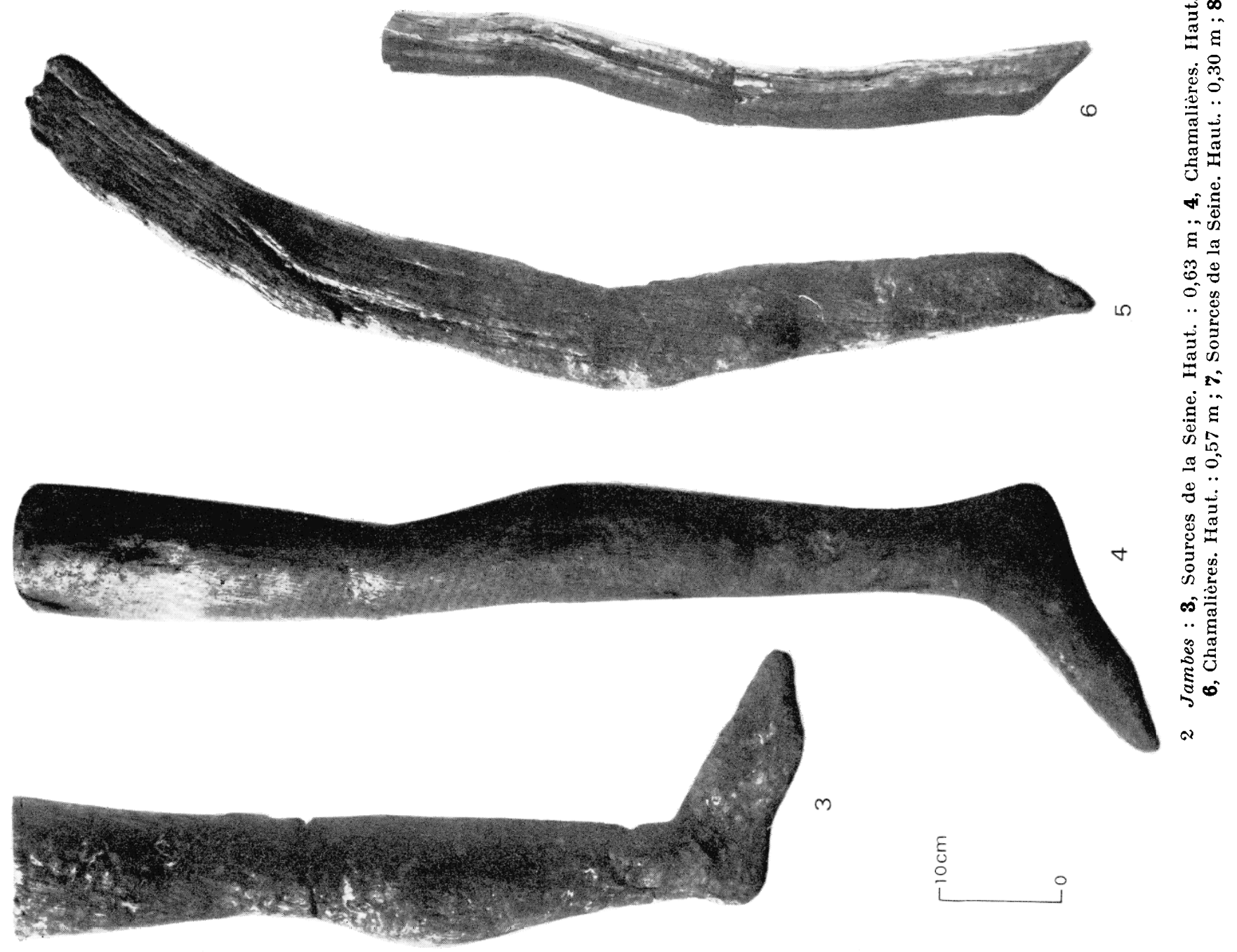
de la Seine ${ }^{21}$; en revanche, le poignet de la manche dessiné relevé au-dessus de la main paraît propre à l'Auvergne (fig. 9, 52). I)e même, une très grande partie des bras de Chamalières tiennent une offrande ronde entre le pouce et l'index (comme le ${ }^{0}$ 53, fig. 9), alors qu'un seul aux Sources de la Seine a la main posée sur une boule, par ailleurs beaucoup plus volumineuse ${ }^{22}$. Enfin, l'un des bras de Chamalières est incontestablement porteur d'une anomalie pathologique ${ }^{23}$, une grosseur à l'aisselle, alors qu'aucun bras de la Seine ne présente une trace de maladie quelconque. Ces différences typologiques, jointes à la disproportion des nombres d'ex-voto entre les deux sites, ne facilitent pas la comparaison stylistique.

Il est cependant possible, là encore, d'effectuer des rapprochements ponctuels entre des ex-voto pris deux à deux, par exemple pour la forme générale d'un bras sans détail musculaire et la représentation des doigts joints bien dessinés, ainsi que pour la qualité de son exécution et la correction de ses proportions (fig. 3, 9 et $\mathbf{1 0}$ ), ou pour l'utilisation de la courbe naturelle d'une branche peu retouchée dont le dessin évoque le coude, avec une main sur laquelle scul le pouce est individualisé (fig. 3,11 et 12). D'autres ont en commun une main presque rectangulaire ${ }^{24}$, ou anguleuse avec un petit pouce pointu dégagé sur le côté (fig. 3,13 et 14).

Une fois de plus, si les sculptures des Sources de la Seine trouvent des équivalents dans quelques exemplaires de la Source des Roches, l'aspect général de la majorité des bras de Chamalières est bien différent de celui des bras de la Seine. Les premiers sont beaucoup plus travaillés, avec la volonté de représentation des détails - épaule, galbe du coude, doigts individualisés -, même si le résultat définitif n'est pas très esthétique (fig. $9, \mathbf{5 0}$ à $\mathbf{5 3}$ ) alors que les derniers sont plus simples et beaucoup moins élaborés.

Les troncs, torses et bassins sont, comme les membres, des thèmes communs aux deux sanctuaires (fig. 3); mais ils n'ont pas été compris et interprétés de la même façon dans les deux cas, ct le "découpage ", notamment, n'a pas été fait de la même manière. Ainsi, les troncs féminins représentés en Bourgogne depuis le cou jusqu'au bas des hanches n'existent pas en Auvergne. En revanche, la plaque rectangulaire portant deux seins en relief des Sources de la Seine est très comparable aux ex-voto de seins de Chamalières ${ }^{25}$, encore que la planche servant de fond au relief dans le premier cas figure probablement un tronc dont il ne saurait être question à la Source des Roches. Autre différence, il existe aussi à Chamalières des représentations d'un sein isolé ou de seins groupés par trois qu'on. ne trouve qu'en pierre en Bourgogne.

Quant aux troncs masculins des Sources de la Seine qui comprennent le haut du corps, des épaules au départ des cuisses, ils n'ont pas d'équivalent exact à Chamalières; on peut seulement rapprocher l'un d'entre eux, coupé du cou au niveau de la ceinture, de quelques bustes longs de Chamalières ${ }^{26}$, mais ces derniers comprennent aussi la tête et peuvent donc avoir une signification différente.

Il en va de même pour les bassins : indépendamment de celui qui n'est que la figuration d'un sexe en fort relief, avec une hernie, sur une plaque hexagonale ${ }^{27}$ et que l'on doit considérer comme une exception, ils ont tous aux Sources de la Seine les jambes coupées à mi-cuisses, tandis qu'à Chamalières les jambes sont représentées jusqu'à l'extrémité des pieds et posées sur un socle (fig. 3 , 15 et 16$)$.

Si le découpage n'est pas le même, en revanche les troncs et les bassins des deux dépôts peuvent être représentés vêtus ou non - plus souvent vêtus à Chamalières, plus souvent nus à la Seine -, et avec ou sans l'indication du sexe. La ceinture parait avoir eu dans les deux cas également une impor-

21 Ibid., op. cit., no 263.

22 Ibid., op. cit., n० 266.

23 Musée Bargoin no 152.

24 S. Deyts, op. cit., no 256, ol Musée Bargoin no 159.
25 Ibid., op. cil., no 67, et Musée Bargoin no 186.

26 Ibid., op. cit., n० 72 ; inédits à Chamalières.

27 Ibid., op. cit., no 73 . 


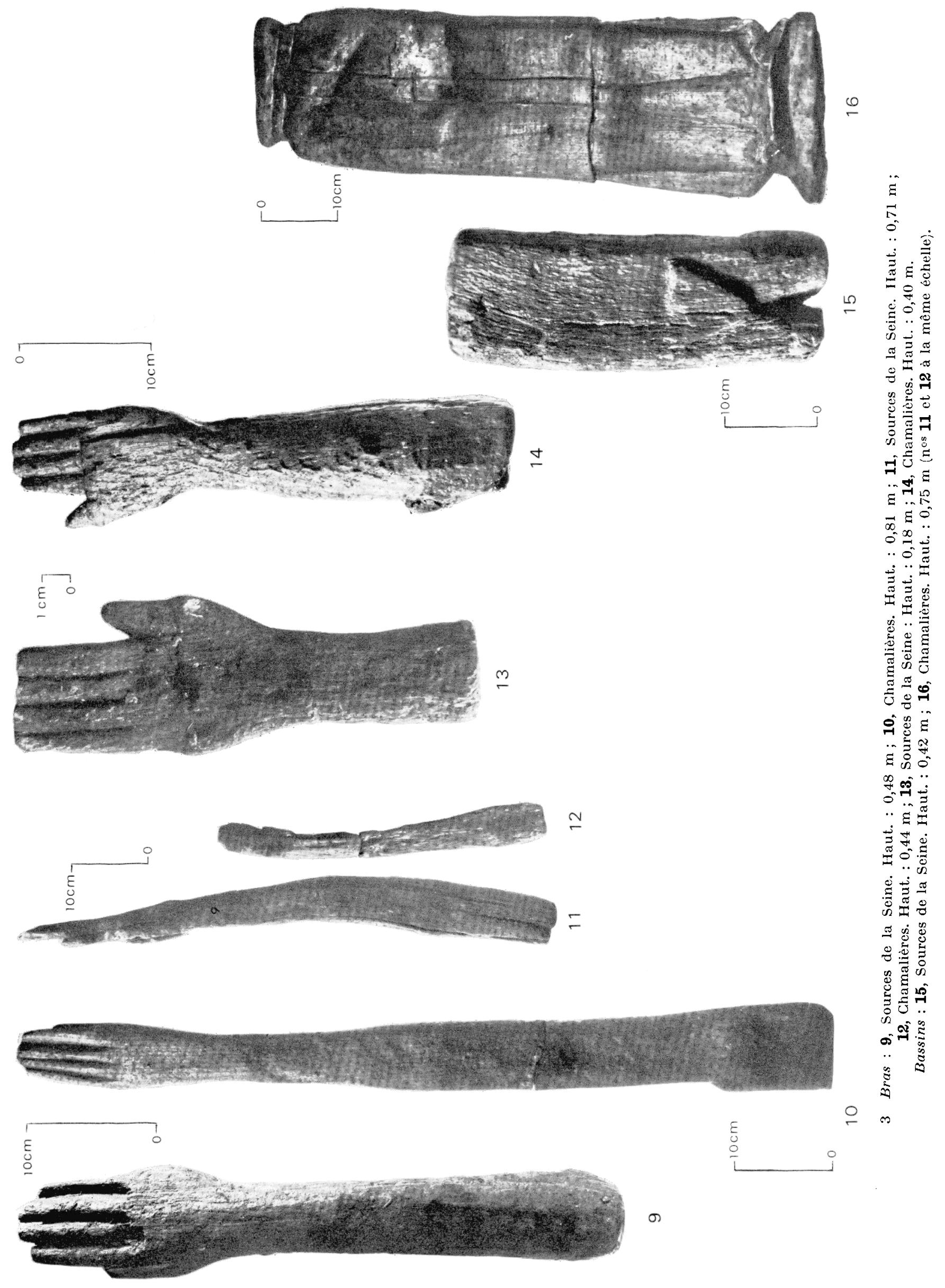


tance particulière : c'est ainsi qu'elle est toujours représentée à C'hamalières sur les bassins vêtus alors qu'on ne la voit jamais sur les personnages représentés en pied, et qu'clle apparaît à la Seine même sur des troncs nus.

Les comparaisons stylistiques entre ces séries trop différentes dans la forme ne peuvent être significatives, mais les rapprochements que l'on peut accomplir montrent cependant que les ex-voto de Chamalières sont dans l'ensemble beaucoup plus évolués et apparaissent comme de véritables sculptures, stylisées certes, mais aussi bien finies que celles des personnages en pied, alors qu'aux Sources de la Seine, ce ne sont que des schématisations frustes et maladroites pour lesquelles le signifié l'emporte à l'évidence sur le signifiant, phénomène qui n'apparaît que rarement à Chamalières.

Au contraire, les planches anatomiques, ex-voto caractéristiques, on l'a vu, de la statuaire de bois, sont étonnamment proches d'un site à l'autre (fig. 4). Dans les deux cas il s'agit de la représentation d'organes internes très schématisés, dont l'identification exacte est difficile, mais sur lesquelles on retrouve les mêmes éléments : crosse de l'aorte, cœur, poumons bilobés, masse abdominale, reins. Lit les deux dépôts ont en commun d'avoir livré des planches anatomiques superposées sur une même plaquette trois aux Sources de la Seine, deux à Chamalières (fig. 4, $\mathbf{1}^{\text {ty }}$ et 18), ce qui montre bien la communauté d'esprit entre les pèlerins des deux sanctuaires. Mais la série des représentations de ce type est beaucoup plus importante et diversifiée en Bourgogne qu'en Auvergne (53 contre 15), et comprend en particulier un type montrant la cage thoracique fermée et les côtes ${ }^{28}$ que l'on ne trouve pas à (Chamalières. De même, si l'on compare les styles des deux séries, il existe aux Sources de la Seine des représentations en ronde bosse, dont une avec la tête surmontant les organes ${ }^{29}$, qui n'existe pas à la Source des Roches.

Cependant, si l'on exclut ces exceptions, l'impression générale que l'on retire de la comparaison entre les planches anatomiques provenant des deux gisements, est celle d'une presque similitude : les organes internes sont représentés en léger relief sur un fond plat, très stylisés, jamais identiques, mais étrangement proches. Par exemple, deux d'entre elles ${ }^{30}$ reproduisent les mêmes thèmes - trachée, poumons bilobés, cœur, diaphragme, masse indifférenciée volumineuse du foie, de l'estomac ou des intestins, et reins -, dans un style identique, la plupart des organes en relief, et certains découpés - la trachée à la Seine, les reins à Chamalières - . Deux autres planches ${ }^{31}$ représentent le cœur, les poumons, et la masse abdominale sur des planchettes de dimensions similaires, peu détaillées, avec des volumes arrondis et modelés. Dans un autre cas (fig. 4, 19 et 20) la forme des organes est semblable, bien que la planche des Sources de la Seine soit beaucoup moins détaillée, et les masses en relief sont découpées sans support de plaquette. Pour d'autres encore (fig. 4, 21 et $\mathbf{2 2}$ ), les ressemblances d'exécution l'emportent sur celles des thèmes, et les organes présentent une même facture aplatie, sans aucun modelé, arrondie à la Seine, anguleuse à Chamalières. Enfin, c'est entre les deux planches portant des représentations multiples (fig. $4, \mathbf{1}^{\text {ty }}$ et 18) que les similitudes sont les plus accomplies : à l'analogie du phénomène de répétition s'ajoute une étonnante ressemblance dans la stylisation des organes; seule la représentation de la trachée diffère légèrement mais le schéma général d'ensemble est identique.

S'il n'existait pas certains thèmes propres aux Sources de la Seine, on pourrait donc considérer qu'il y a identité absolue entre les productions des deux sites. Cette catégorie d'ex-voto est réellement celle où les similitudes sont les plus fortes, ce qui suggère qu'il y a eu échange de modèles dans un sens ou dans l'autre. Mais c'est un phénomène qui peut s'expliquer aussi par la destination uniquement votive de ces objets qui n'ont aucun point commun avec la grande statuaire, contrairement aux sculptures de bustes et de personnages entiers bien sûr, mais même aux sculptures des membres qui peuvent être assimilés par l'artisan à des parties de statues en pied. Enfin, le fait que les organes

28 Ibid., op. cil., nos 159 à 163 , par exemple.

29 Ibid., op. cil., no 199.
30 Ibid., op. cil., no 179 , et Musée Bargoin no 183.

31 Ibid., op. cit., no 184, et Musée Bargoin no 184. 


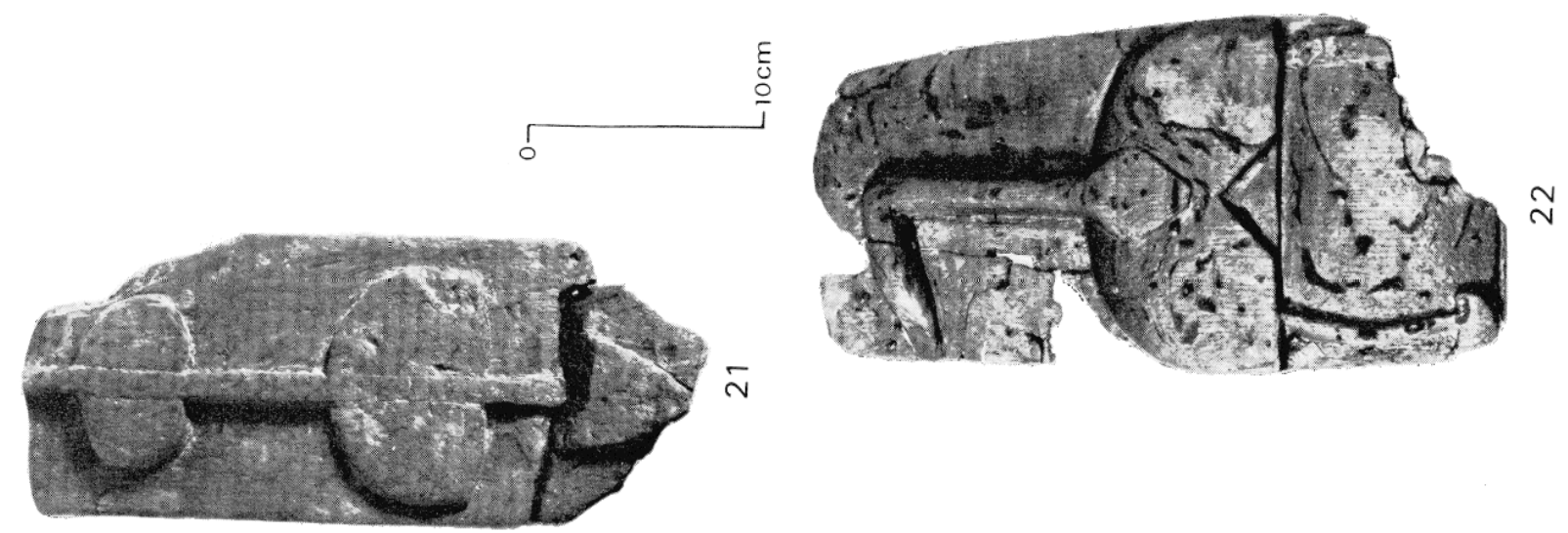

苍曷

I

政

요

\&

品

范
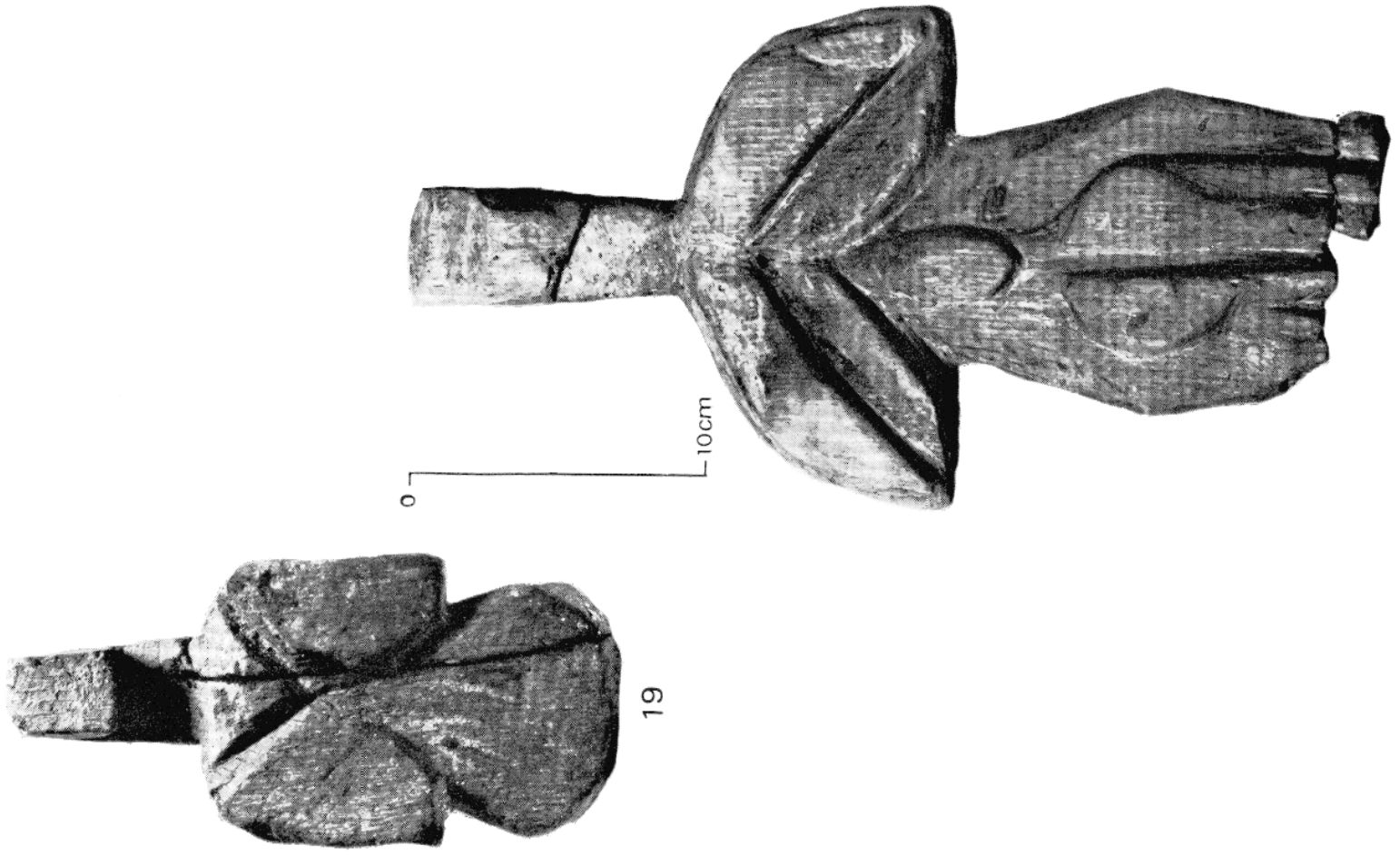

क्षे

घ

ค. ฝิ

ำ

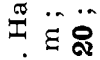

ำ

बं山े

$\cong$ ㅇ.

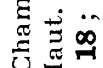

U⿺辶一兀

舟

$\ddot{g})$

\& $\infty$

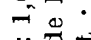

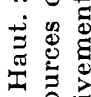

工

केष

政

䑻

Ðึ

苛

품

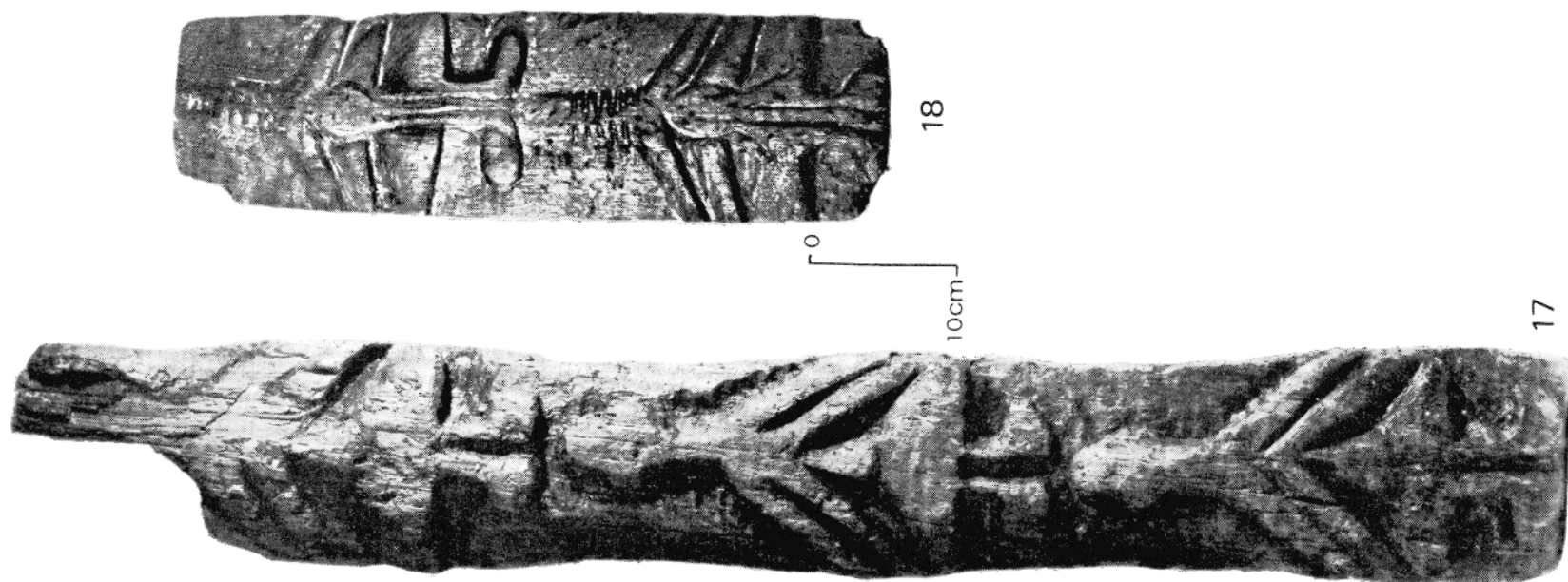

$\ddot{0} \dot{0}$ 
figurent en bas-relief sur des planches aux Sources de la Seine alors que la majorité des autres ex-voto du site sont en ronde bosse, ajoute certainement beaucoup à cette communauté d'aspect.

Les personnages entiers présentent plus d'originalité (fig. 5 et 6 i. Sur les deux sites ils sont représentés debout, de face, les pieds reposant sur un socle généralement plat, exceptionnellement épointé, et les hommes sont beaucoup plus nombreux que les femmes. A Chamalières trois personnages échappent à cette règle : deux femmes assises et un homme à cheval. Ce sont, il est vrai, des pièces exceptionnelles, mais elles n'en constituent pas moins des types d'ex-voto ignorés aux Sources de la Seine. I) même, un bon nombre de pèlerins de Chamalières, un tiers environ, portent des offrandes, alors qu'aucune sculpture de bois de la Seine n'a cette particularité, très répandue par ailleurs sur la statuaire de pierre du mème site. Enfin, il existe en Bourgogne des personnages nus alors que la représentation de la nudité est réservée en Auvergne aux sculptures de bassins; et, tandis qu'aucune anomalie pathologique ne peut être décelée à Chamalières, trois personnages de la Seine ont été interprétés comme des malades, bien que dans les trois cas l'évocation de la maladie soit extrêmement discrète.

Les vêtements aussi sont diflérents, lorsqu'ils sont représentés. Car, dans les deux sanctuaires existent des statues "sans aucun détail vestimentaire apparent" sur lesquelles scule la tête a été détaillée et qui ont un caractère d'ébauches d'où est exclu tout réalisme (fig. 5, 23 et 24). Les ex-voto de Chamalières présentant ces caractéristiques sont en général de dimensions beaucoup plus réduites que les autres, et c'est ainsi qu'une statuette haute de $13 \mathrm{~cm}$ (fig. 5, 24) peut être comparée à une statue des Sources de la Seine qui mesure $122 \mathrm{~cm}($ fig. 5,23$)$ : elles ont la même forme de tête au sommet presque plat, avec un visage rectangulaire aux traits schématisés par incisions sans souci de réalisme, un cou tronconique mal dégagé, un corps cylindrique aux épaules à angle droit de même largeur que la tête, et retouchè seulement par un coup d'outil oblique sur la partie antérieure à l'emplacement de l'encolure. Deux autres statues, de tailles également dissemblables ${ }^{32}$ ont en commun la frange de cheveux courts sur le front, la représentation du menton plaqué sur le cou, la forme des épaules anguleuses, et la platitude du corps. Malgré ces similitudes, il est clair que les statues des Sources de la Seine sont plus belles et plus détaillées du fait de leurs dimensions et pourraient effectivement être des ébauches ${ }^{33}$ car l'une d'elles a des oreilles et un menton (fig. 5, 23) et l'autre un visage sommairement modelé alors qu'il est totalement exclu que les statuettes de Chamalières ne soient pas achevées.

Parmi les personnages vêtus, un certain nombre d'hommes aux Sources de la Seine portent une lunique tombant en pointe sur le côté ${ }^{34}$. Ils ressemblent beaucoup à ceux de Chamalières sur lesquels on peut reconnaître le manteau agrafé sur l'épaule qui s'arrête obliquement à mi-corps et tombe en formant un long pan sur le côté, même s'il n'est pas assuré qu'il s'agisse exactement du même vêtement. On peut ainsi rapprocher (fig. 5, 25 et 26) deux personnages qui sont par ailleurs dans les deux cas en mauvais état, mais qui conservent des silhouettes générales comparables : tête allongée, corps plat peu détaillé, et jambes au galbe accusé. Deux autres ${ }^{35}$ ont en commun le mouvement oblique du vêtement sur les jambes également modelées, celui du tissu sur l'épaule droite, et le geste du personnage, bien que des détails apparaissent à Chamalières - les doigts, et le drapé plus net -, qui font défaut à la Seine, et que cette dernière sculpture ait plus de relief. Enfin, il existe une intéressante analogie de costumes entre deux pèlerins aussi exceptionnels l'un que l'autre ${ }^{36}$, qui portent une tunique s'ouvrant sur le devant en forme de "jaquette», et ont, de surcroît, la même coiffure à godrons, très fréquente en Auvergne, mais unique sur les figurations de personnages entiers en Bourgogne.

35 Ibid., op. cit., no 18, et Musée Bargoin no 35.

36 Ibid., op. cit., no 16, et Musée Bargoin no 33. 

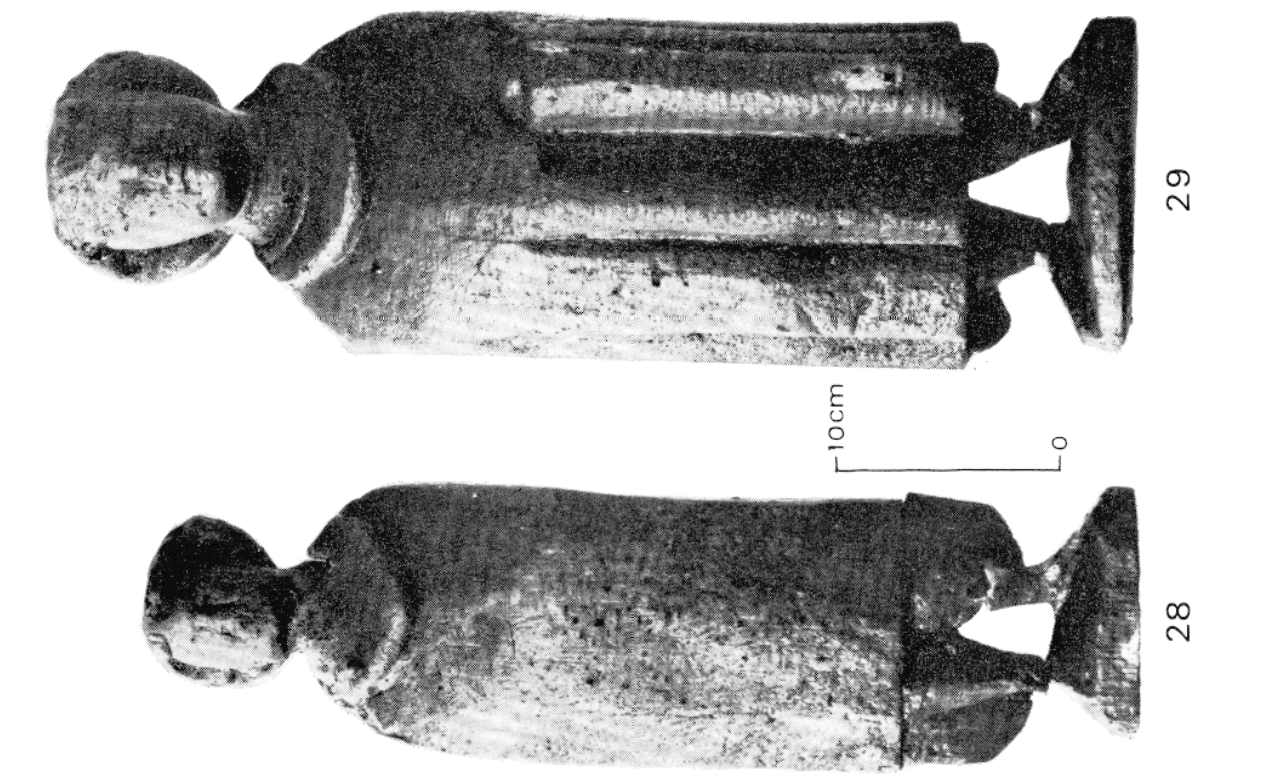

$\ddot{g}$
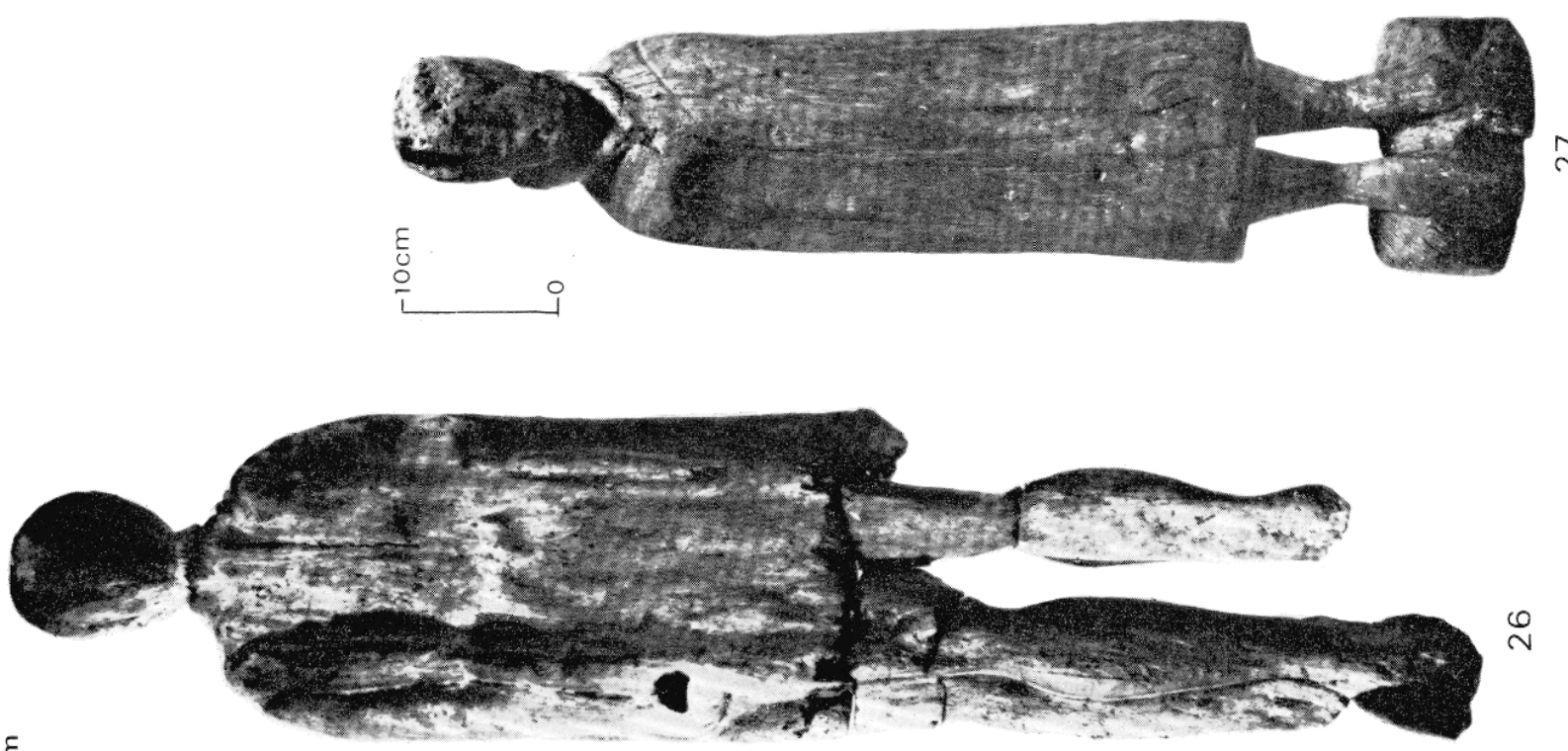

$\stackrel{5}{i}$

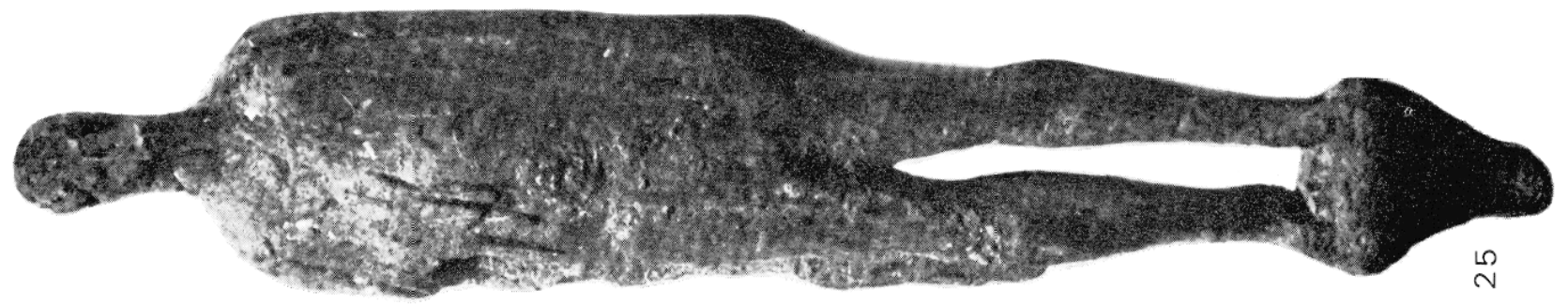

욜

寻芆俩

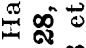

远

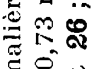

है.

نี

ลี

ह्व

然

‥

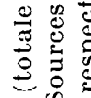

요

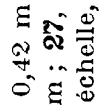

هं

猔:

造泀

音

$\exists$

घี

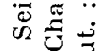

要

응

की

跣

क. . ह

สํ

ชื

छ

苋若

$= \pm 0$

过

芌嵒离
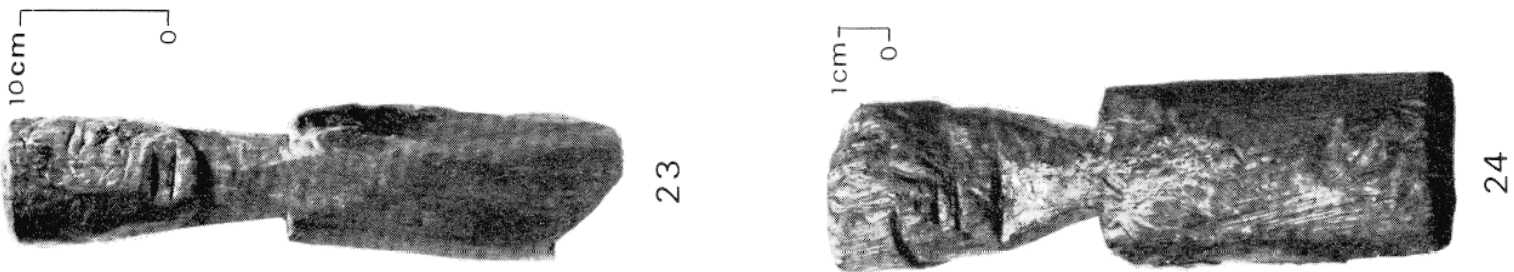
Cependant le plus répandu des vêtements reste la pèlerine, portée par la majorité des personnages masculins des Sources de la Seine et par $90 \%$ de ceux de Chamalières, un nombre suffisant par conséquent pour permettre des comparaisons. Ces pèlerins sont représentés debout, vêtus de la cape des voyageurs avec un gros bourrelet marquant l'encolure, mais ceux de Chamalières sont moins diversifiés, n'ont pas le capuchon relevé sur la tête comme en Bourgogne ni figuré rabattu dans le dos, sauf de façon exceptionnelle. Ils forment une série très stéréotypée, par opposition à la diversité de ceux des Sources de la Seine, plus monotone mais aussi mieux finie, comme si la spécialisation dans ce type de traitement avait entraîné un perfectionnement de la technique.

Ainsi lorsque l'on compare deux pèlerins d'origines différentes (fig. 5), 27 et 28) choisis parmi les plus proches, on note qu'à Chamalières la tête est mieux dégagée, le visage plus rond, séparé du corps par un cou qui n'existe pas aux Sources de la Seine, et que la coiffure est non seulement indiquée par un volume bien différencié de celui du visage, mais détaillée et travaillée en godrons. La rigidité du vêtement qui cache tout détail anatomique du corps rectangulaire et des bras se retrouve dans les deux cas, mais à Chamalières deux petits pans en retrait dans le dos de part et d'autre des jambes, dénotent un souci du détail inconnu aux Sources de la Seine, bien qu'ils soient difficiles à interpréter et n'ajoutent rien à la qualité artistique de la sculpture. Les jambes, en revanche, présentent dans les deux cas la même forme triangulaire, la même absence de modelé, et se terminent par des pieds schématisés posés sur un socle.

La statuette de Chamalières précédemment décrite (fig. 5,28 ) reste donc très stylisée mais elle apparaît déjà plus élaborée que celle des Sources de la Seine, et cette qualité de travail est encore plus sensible si l'on considère une autre stèle mieux achevée (fig. 5, 29), sur laquelle les traits du visage ont été modelés avec précision et le vêtement traité avec soin : la pèlerine tombe en formant plusieurs plis verticaux en fort relief, dont l'un plus volumineux que les autres suggère le mouvement du bras gauche plié sous la pèlerine pour présenter une offrande ronde qui apparaît au-dessus du pli; une deuxième encolure arrondie est visible derrière celle du manteau, et les petits pans descendant de part et d'autre des jambes ont un aspect réaliste évoquant la souplesse du tissu. Ce personnage pourrait être comparé aux pèlerins sculptés dans la pierre des Sources de la Seine ${ }^{37}$ qui ont eux aussi une facture plus achevée que celle des statues de bois.

La différence de style entre les personnages provenant des Sources de la Seine et de Chamalières peut également s'apprécier par la comparaison entre deux pèlerins, loujours vêtus de cette même cape, mais qui constituent par leur originalité sur l'un et l'autre site des exemplaires isolés ${ }^{38}$ : alors que l'ex-voto bourguignon est une cuvre amusante et vigourcuse d'où est exclu tout réalisme mais qui témoigne d'une technique bien maîtrisée, la même technique a permis en Auvergne la réalisation d'une sculpture beaucoup plus évoluée. Les deux pèlerins ont en commun une tête trop grosse, des traits stylisés et peu saillants, et la même forme du corps approximative avec un vêtement schématisé; mais alors que la statue des Sources de la Seine est linéaire et caricaturale, celle de Chamalières est moins disproportionnée, avec des volumes modelés et une stylisation épurée.

En revanche, deux personnages représentés l'un nu en Bourgogne et l'autre vêtu en Auvergne ${ }^{39}$ se ressemblent réellement, non seulement par le contraste entre le corps rectiligne et la tête élaborée, répondant au fameux principe de l'« exaltation de la tête $\|^{40}$, mais aussi par le traitement du visage allongé, de la coiffure en calotte, des yeux globuleux sous les arcades sourcilières proéminentes, de la bouche aux commissures tombantes, et du nez bien dessiné.

Pour les femmes, la comparaison est moins significative car les Sources de la Seine n'ont livré qu'un petit nombre d'ex-voto féminins, dont trois seulement vêtus. On trouve certes à Chamalières

37 Ibid., op. cil., pl. LXXXII a et e.

38 Ibid., op. cil., n" I et Musée Bargoin n" 15.

39 Ibid., op. cil., n" 38 , el Wusée Baryoin n" 13.

40 P. JАмвнысатs, Lexallation de la lele dans la pensée el dans larl des Celles, Bruges, 1954. Expression reprise par S. DErTs, op. cil., nolamment p. 152, a propos de plusieurs sculphures des sources der la seine. 

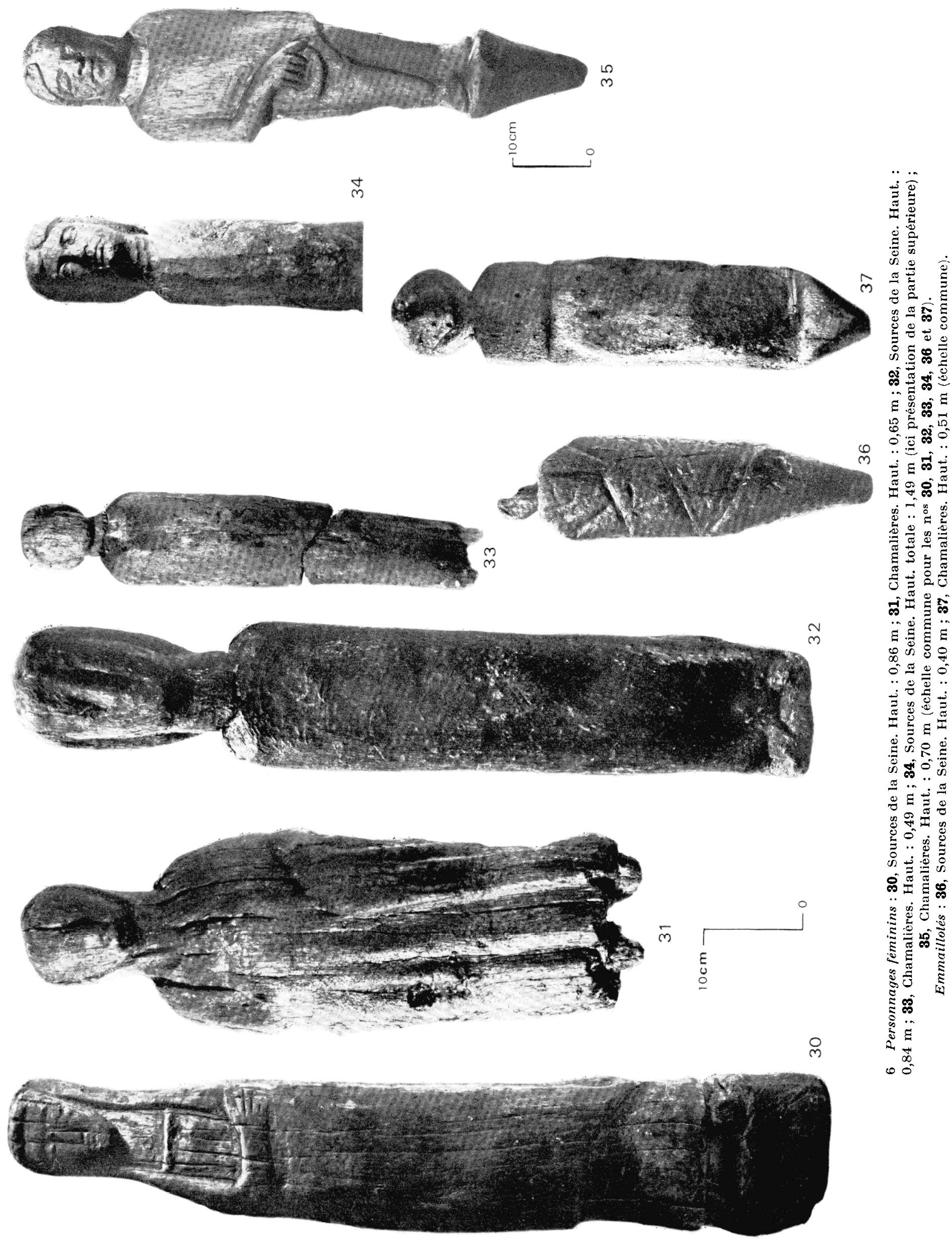
les deux types de vètements bourguignons, manleau drapé avec encolure carrée et tunique entièrement plissée, mais sur des exemplaires isolés, alors que le costume le plus répandu en Auvergne est de loin le manteau agrafé sur l'épaule, et, pour quelques unités, la pèlerine indigène semblable à celle des hommes. Si l'on ne compare que les quelques femmes vêtues de la même manière sur les deux sites, il est évident que le manteau drapé avec encolure carrée porté par une belle statue des Sources de la Seine (fig. 6, 30), anguleuse et géométrique, est traité dans un tout autre style à Chamalières sur une statue en chêne elle aussi (fig. 6,31 ) : tout est lignes droites sur la première - forme de la tête, du bras, du vêtement, des pieds -, et tout est courbes sur la seconde - visage rond, tissu souple, plis ondulés, geste du bras -.

Sur une autre statue féminine à la tunique entièrement plissée des Sources de la Seine ${ }^{41}$. on peut noter, au contraire, en dépit de son mauvais état de conservation, un effet de divergence qui témoigne d'un dessin plus élaboré qu'à Chamalières où les plis sont rectilignes.

Mais il existe aussi sur les deux sites des femmes dont le seul détail vestimentaire apparent est la bordure inférieure du vêtement au-dessus des pieds. C'est beaucoup plus fréquent aux Sources de la Seine où cinq personnages sur neuf sont dans ce cas, qu'à Chamalières où il ne s'agit que d'exemplaires isolés et le plus souvent abîmés. Il est donc périlleux de prétendre les comparer, mais il est certain que ces sculptures (fig. 6, 32 et 33) relèvent du même esprit et s'apparentent peut-être aux suivantes, dans la manifestation, une fois encore, de l'exaltation de la tête.

Cette exaltation célébrée aux Sources de la Seine par une statue haute de $1,49 \mathrm{~m}$ pour une tête de $10 \mathrm{~cm}$ très travaillée, au-dessus d'un corps longiligne à peine dégrossi (fig. 6, 34), trouve son équivalent à Chamalières dans deux types de représentations très différentes : d'une part de grandes stèles au corps très long et très plat surmonté d'une tête relativement petite et beaucoup plus détaillée ${ }^{42}$; d'autre part le portrait d'une vieille femme dont le corps minuscule et maladroitement sculpté contraste avec la tête très élaborée qui ressemble trait pour trait à celle de la grande statue de la Seine - même chevelure longue en masse séparée par une raie médiane, mêmes yeux globuleux et nez épais aux narines dessinées, même bouche aux lèvres charnues, même menton fort (fig. 6,35$)$--

La dernière catégorie de personnages entiers offerts en ex-voto était celle des emmaillolés (fig. 6). Ils sont aussi rares sur un site que sur l'autre : trois à Chamalières, un seul aux Sources de la Scine, alors qu'ils sont assez fréquents dans la statuaire de pierre. Leur représentation participe de la même stylisation dans les deux cas (fig. $6, \mathbf{3 6}$ et $\mathbf{3 7}$ ) : la tête est petite, le corps rectangulaire caché par le maillot, les épaules obliques, ct l'extrémité inférieure taillée en pointe pour schématiser l'annincissement de cette partie du vêtement que l'on constate sur les ex-voto de pierre du même type, aussi bien en Gaule qu'en Italie.

On peut donc trouver des similitudes de thème ou de style pour chaque type de personnage entier, homme, femme, ou emmailloté, provenant des deux sites, mais elles demeurent limitées puisqu'clles procèdent surtout de comparaisons ponctuelles portant souvent sur des sculptures de Chamalières en chêne, et qu'il n'existe pas aux Sources de la Seine de stèles féminines semblables à celles de la série majoritaire de Chanalières, qui sont en hêtre, sculptées en faible relief comme leurs homologues masculins et vêtues le plus souvent d'un manteau agrafé sur l'épaule au-dessus d'une tunique longue (fig. 10, $\mathbf{5 6}$ et 57). Avec celles des Sources de la Seine, ces dernières, nettement plus détaillées, n'ont en commun que quelques traits dénotant cependant une certaine concordance d'esprit : l'attitude frontale, le canon généralement court. l'étude anatomique réduite, et la schématisation des drapés.

La dernière série d'ex-voto est celle des têtes et des bustes (fig. 7 et 8). Ces représentations sont fréquentes sur les deux sites mais on ne trouve aux Sources de la Seine que des têtes coupées sous le cou 

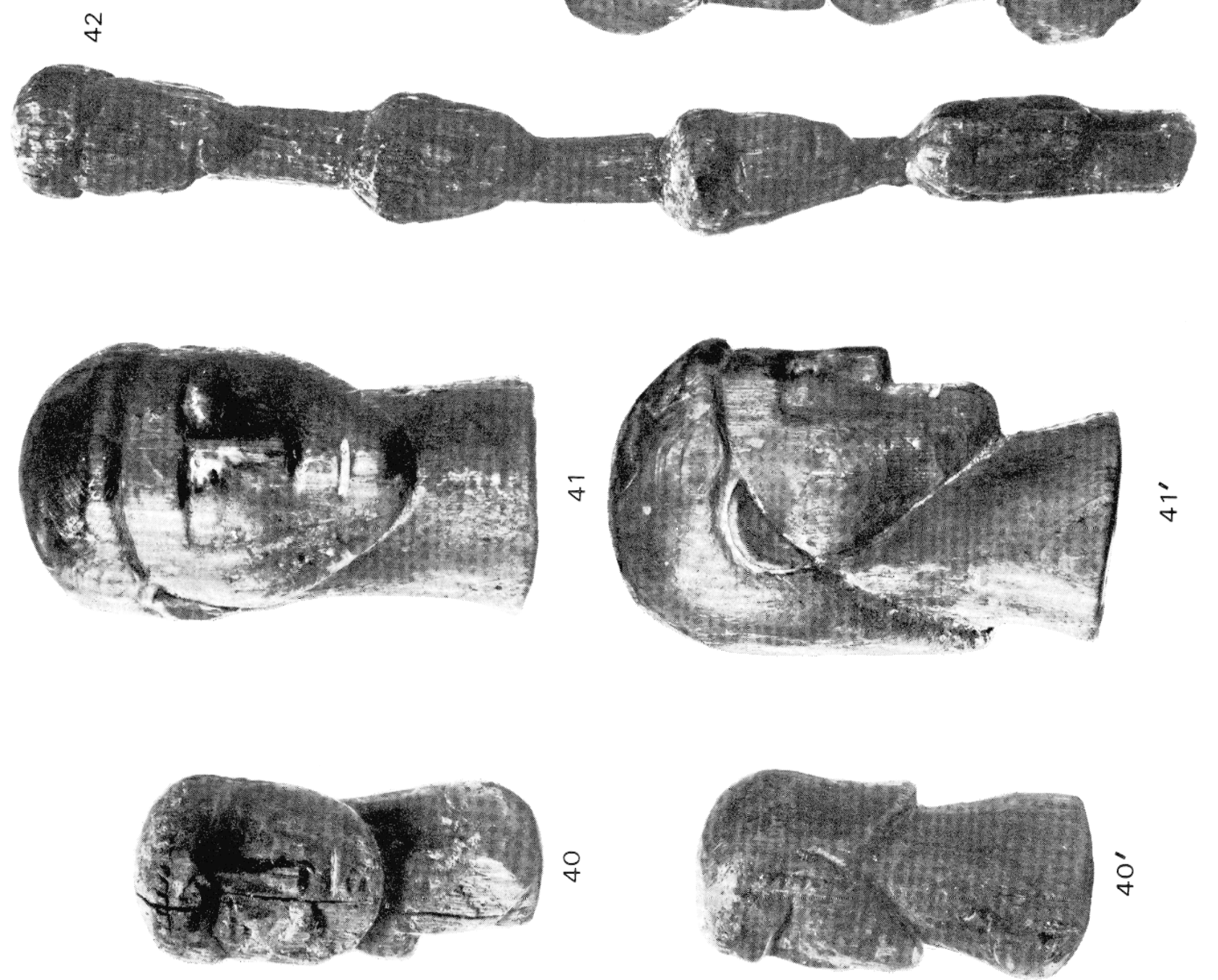

药

范

융요

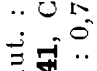

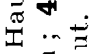

घ

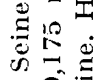

a 0.

요

过

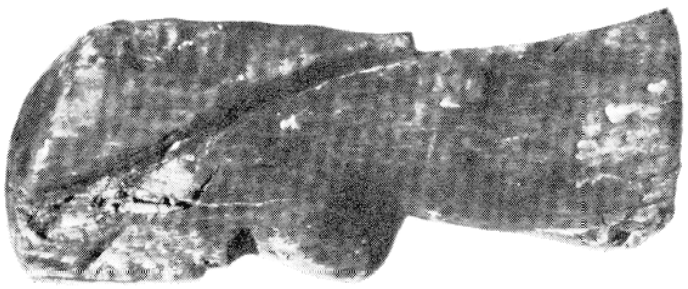

के

ज.

我苗

녕

ڤั

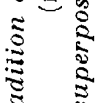

\&

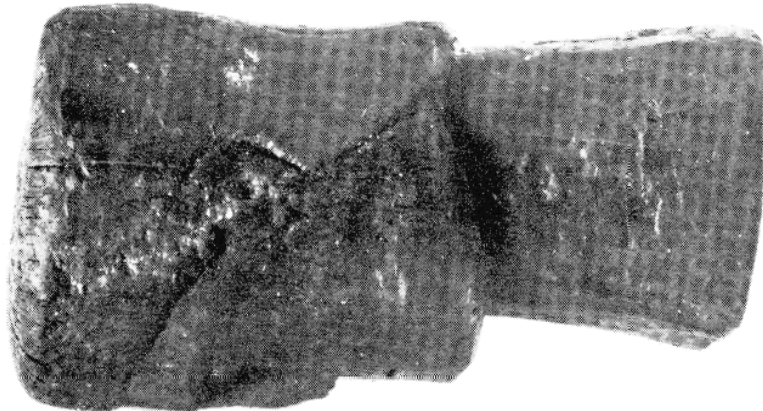

$\stackrel{\infty}{m}$

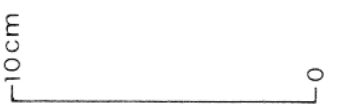


à une exception près, alors qu'à Chamalières ce sont surtout des bustes comprenant le haut des épaules. Quatre têtes des Sources de la Seine ont aussi la particularité, qui apparaît une fois à la Source des Roches, d'avoir un tenon fixé à leur base $e^{43}$. Et il existe à Chamalières deux types inconnus en Bourgogne : deux têtes destinées à être fixées sur un buste passe-partout, et quelques têtes en relief sur un médaillon ou une plaquette ${ }^{44}$. Enfin, alors qu'aucune affection pathologique n'est patente sur les têtes de la Seine, un personnage de Cilhamalières dont une main est plaquée sur l'oreille et l'autre sur la bouche représente certainement un sourd-muet ${ }^{45}$.

Quant aux coiffures, elles se ressemblent mais présentent des variantes : celle des hommes aux Sources de la Seine avec la masse des cheveux ramenés en frange sur le front et encadrant le visage par une courbe continue qui tombe en arrondi sur la nuque ${ }^{46}$ est aussi celle des têtes de Chamalières, qu'elles soient frustes ou de belle qualité, et vraisemblablement également celle des bustes, mais avec un traitement très différent puisque dans ce cas les mèches sont stylisées par des godrons. Pour les femmes, la comparaison est malaisée à cause du plus petit nombre d'exemplaires; si l'on peut cependant noter la similitude entre la coiffure mi-longue à godrons d'une femme de la Seine (fig. 8, 46) et celle de la majorité des bustes féminins de Chamalières, bien des coiffures sur ce dernier site sont beaucoup plus élaborées, avec diadème et voile ou cheveux sur les épaules, alors qu'il n'existe de comparable qu'une amorce de diadème aux Sources de la Seine ${ }^{47}$. Inversement, il n'existe pas à Chamalières de coiffure relevée à l'arrière et déportée sur le côté, ou avec un chignon bas ${ }^{48}$, mais la plupart des sculptures de ce sanctuaire ont un revers plat qui ne permettait pas ce genre de représentation.

Autrement dit, tous les types des Sources de la Scine existent en Auvergne, à quelques variantes de coiffure près, mais la série de Chamalières est plus diversifiée, et la catégorie la plus abondante en ce lieu, celle des bustes, n'est représentée en Bourgogne que par un exemplaire isolé.

En revanche, les figurations très originales de lêles superposées (fig. 7, 42 et 43) qui sont caractéristiques de la sculpture de bois et ne sont connues à ce jour que dans ces deux sanctuaires, sont plus nombreuses aux Sources de la Seine puisqu'on y compte dix-huit représentations contre trois à Chamalières. Elles y sont généralement groupées par trois - et c'est aussi le cas pour deux exemplaires de la Source des Roches -, mais également par deux quatre fois. Il existe enfin en Bourgogne deux groupes de têtes opposées qui sont absents en Auvergne. Incontestablement c'est un thème qui a été développé avec plus de succès aux Sources de la Seine.

Si l'on excepte cependant cette série de têtes superposées, il est clair que, de même que les types, les styles des têtes et des bustes sont beaucoup plus variés à Chamalières qu'aux Sources de la Seine où la production est assez homogène, et où il y a moins d'écart entre les sculptures les plus frustes et les sculptures les plus belles. Et si l'on peut classer les ex-voto de l'un et l'autre site dans des catégories stylistiques qui se correspondent, les proportions et l'aspect général des deux séries sont très dissemblables : le style le plus courant aux Sources de la Seine est celui qui a été qualifié de "tradition celte "49, et le style le plus courant à Chamalières est celui des bustes en hêtre à coiffure à godrons (fig. 11, 61 à 64). Entre les deux, il existe un grand fossé, mais il est néanmoins possible d'effectuer des rapprochements ponctuels entre certains ex-voto pris isolément.

En dehors des deux très belles têtes appartenant à la "grande statuaire "50 et des représentations très sommaires que forme le groupe des têtes superposées, toutes les têtes des Sources de la Seine ont

43 S. Deyts, op. cit., nos $82,83,129$, 130. Exemplaire inédit à Chamalières.

44 Têtes sur médaillons : Informations dans Gallia, 27, 1969,2 , p. 33 , no 24 , et Musée Bargnin no 92. I.es autres types ne sont pas publiés.

45 Inédit.
46 S. DEYTs, op, cit., p. 136.

47 Ibid., op. cil., no 104.

48 Ibid., op. cil., nos 10 ; et 113 .

49 Ibid., op. cit., p. 151-152.

50 Pour reprendre l'expression de s. l)eres, op. cit., p. 152-15:3. 


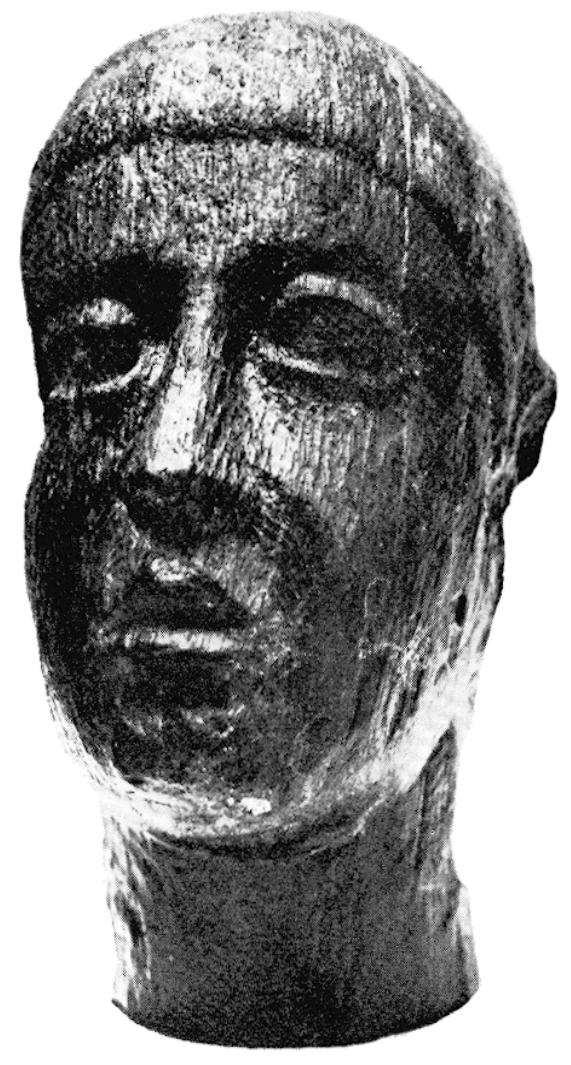

44
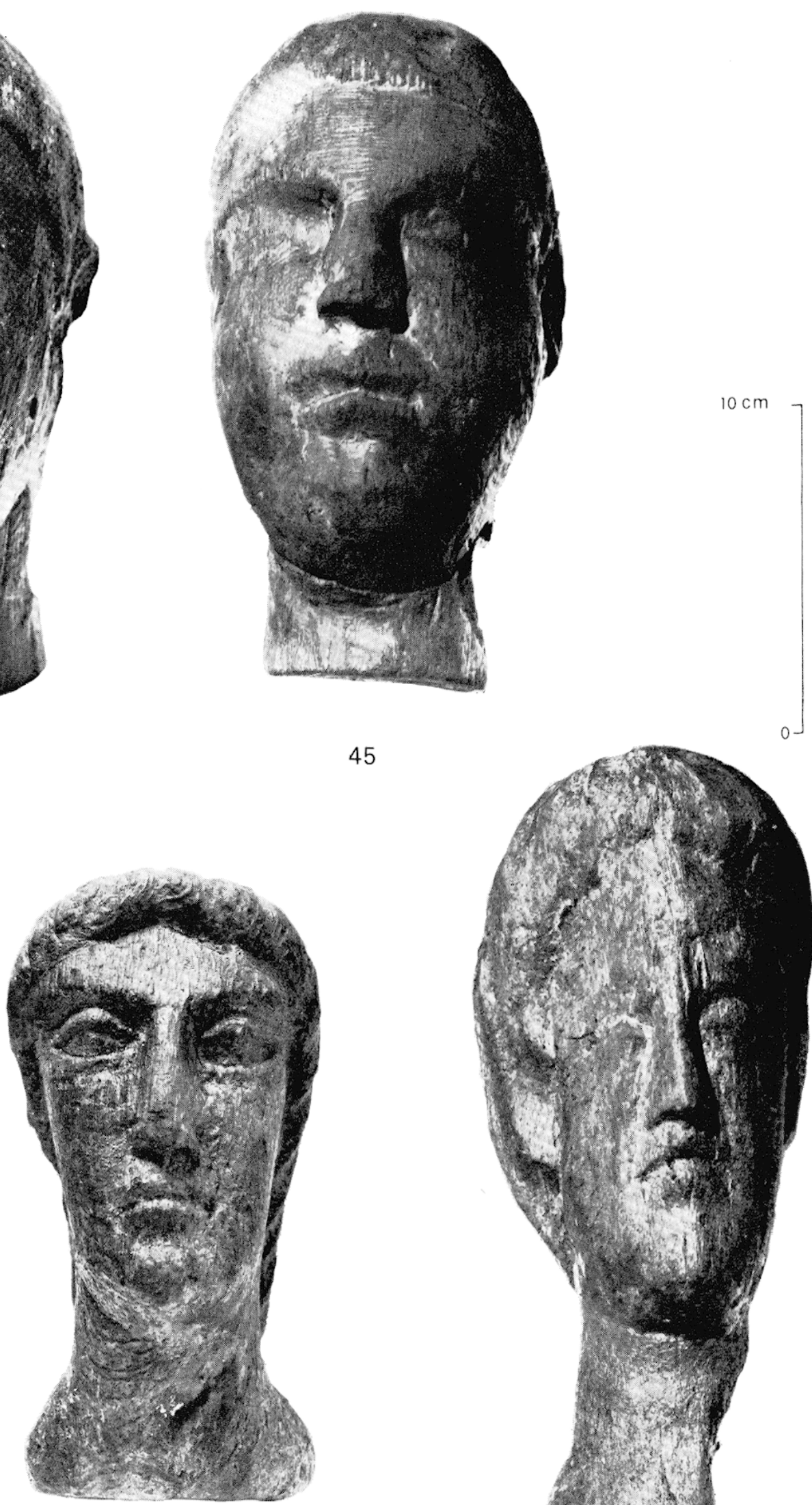

46

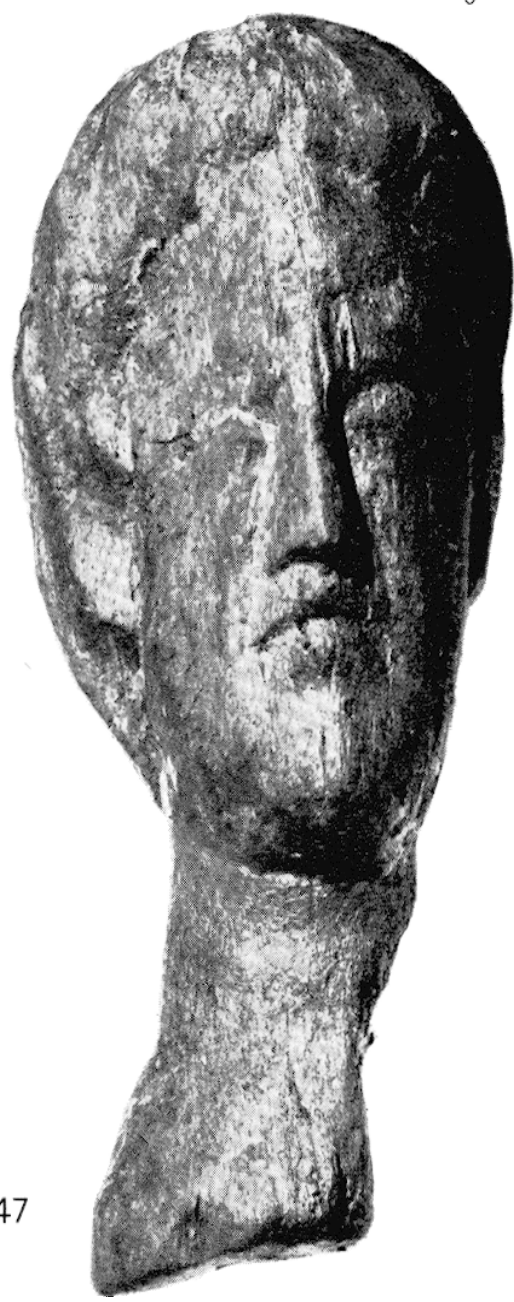

8 Tètes appartenanl à la "grande statuaire " : 44, Sources de la Seine. Haut. : 0,22 m ; 45, Chamalières. Haut. : 0,21 m ; 46, Sources de la Seine. Haut. : 0,20 m; 47, Chamalières. Haul. : 0,27 m (échelle commune). 
donc un style assez proche, "de tradition celte "51, pour lequel il y a des parallèles peu nombreux mais significatifs à Chamalières. Ainsi peut-on rapprocher deux têtes qui ont, vues de profil, la même forme quadrangulaire de la tête, triangulaire du visage et de la chevelure, tronconique du cou, le même nez proéminent, et la même bouche horizontale (fig. 7, 38 et 39). Deux autres présentent les mèmes traits stylistiques, mais en plus élaborés : le visage ovalisé plus rond, les globes oculaires marqués, le nez triangulaire, la bouche plus petite, le pavillon de l'oreille indiqué, les mèches de cheveux stylisées, incisées dans l'épaisseur de la frange à Chamalières, détaillées sur le front en denticules aux Sources de la Seine, et le profil toujours géométrique mais plus réaliste (fig. 7, 40 et 41). Deux dernières enfin, témoignant d'un travail plus sommaire, ont en commun des dimensions réduites, la forme massive de la tête représentée sans cou, la chevelure découpée en calotte, les traits marqués par incisions, le nez en faible saillie, et des traces de coups d'outil encore visibles ${ }^{52}$.

Pour ce qui est des têtes superposées, encore plus frustes, certaines se ressemblent énormément, on l'a vu, d'une région à l'autre, bien que celles de Chamalières soient nettement plus petites. Ial similitude est particulièrement sensible sur deux d'entre elles qui présentent la même succession de têtes ovales aux visages désaxés, sur des cous minces, et les mêmes traits incisés rectilignes sous une lourde frange dissimulant le front (fig. 7, 42 et 43).

Enfin les deux têtes appartenant à la "grande statuaire" des Sources do la Scine ont aussi à Chamalières des équivalents précis, et peuvent être considérées également comme des prototypes préfigurant deux styles de Chamalières qui se sont développés en ce lieu de façon inégale. Ainsi le portrait d'Auvergnat (fig. 8, 45) n'a-t-il rien à envier par son réalisme à son homologue bourguignon (fig. 8, 44) : la forme et les dimensions des deux sculptures sont identiques, le traitement de la chevelure peu volumineuse en calotte dégage de la mème façon des oreilles bien dessinées; le schéma du visiage aux joues pleines avec un gros menton rond et des traits bien modelés est le même à quelques détails près; seuls les yeux sont différents. Cette sculpture, qui donne une impression d'achèvement et de perfection technique, est unique aux Sources de la Seine. A Chamalières on peut lui rattacher d'autres pièces, peu nombreuses certes, mais formant cependant une petite série exceptionnelle : ainsi dans le genre du portrait la femme au torque (fig. 11,58 ) et dans un style beaucoup plus idéalisé deux têtes masculines, l'une toute en rondeurs, l'autre aux lignes étirées en longueur (fig. 11, 59 et 60).

Quant au buste féminin qui est tout aussi exceptionnel aux Sources de la Seine (fig. 8, 46), il est étonnamment proche d'un buste de (hamalières (fig. $8,4{ }^{\text {7y) }}$ ) qui fait lui-même partie de la série très abondante des bustes à coiffure stylisée en godrons. Ils ont en commun lia même forme du visage allongé au menton empâté, encadré de mèches de cheveux rayonnant à partir du sommet du crâne, et formant une courte frange sur le front, le nez droit et la bouche aux commissures tombantes, ainsi que lc même petit buste court, unique à la Seine, presque de règle à Chamalières. Seuls les yeux, là encore, diffèrent : le globe de l'œil dans le premier cas est cerné d'une incision alors qu'il n'y a que la paupière supérieure ainsi délimitée dans le deuxième cas, suivant un traitement très fréquent et caractéristique en Auvergne.

Les autres bustes de Chamalières à coiffure à godrons sont moins proches de ceux des Sources de la Seine, et comme ils sont très nombreux, ils présentent des différences de détail qui varient selon les cas: forme du visage plus arrondie, cou plus allongé, traits plus schématisés, socle plus haut, présence de l'encolure du vêtement, pan de cheveux ou voile sur l'épaule, revers de la sculpture rigoureusement plat (fig. 11, 62 à 64). Mais ils ont, c'est évident, le même modèle, avec des variantes de traitement plus ou moins prononcées, qui ont produit de très belles œuvres lorsque le sculpteur expert dans ce type de réalisation en prenait le temps, et d'autres assez schématisées, vraisemblablement rapidement exécutées dans un souci de rendement.

51 Cf. note no 49 . On peut $y$ inclure aussi celles dont les traits du visage et les details de la chevelure sont effacés mais qui ont les mêmes caractéristiques de dimensions et de composition ínos 116 a 137 du catalogue de s. Deyts y, yui ont ité considerées comme "frustes ou ébauchèes".

52 S. l)ErTs, op. cil., n 85, el Musée Bargoin n" 97. 

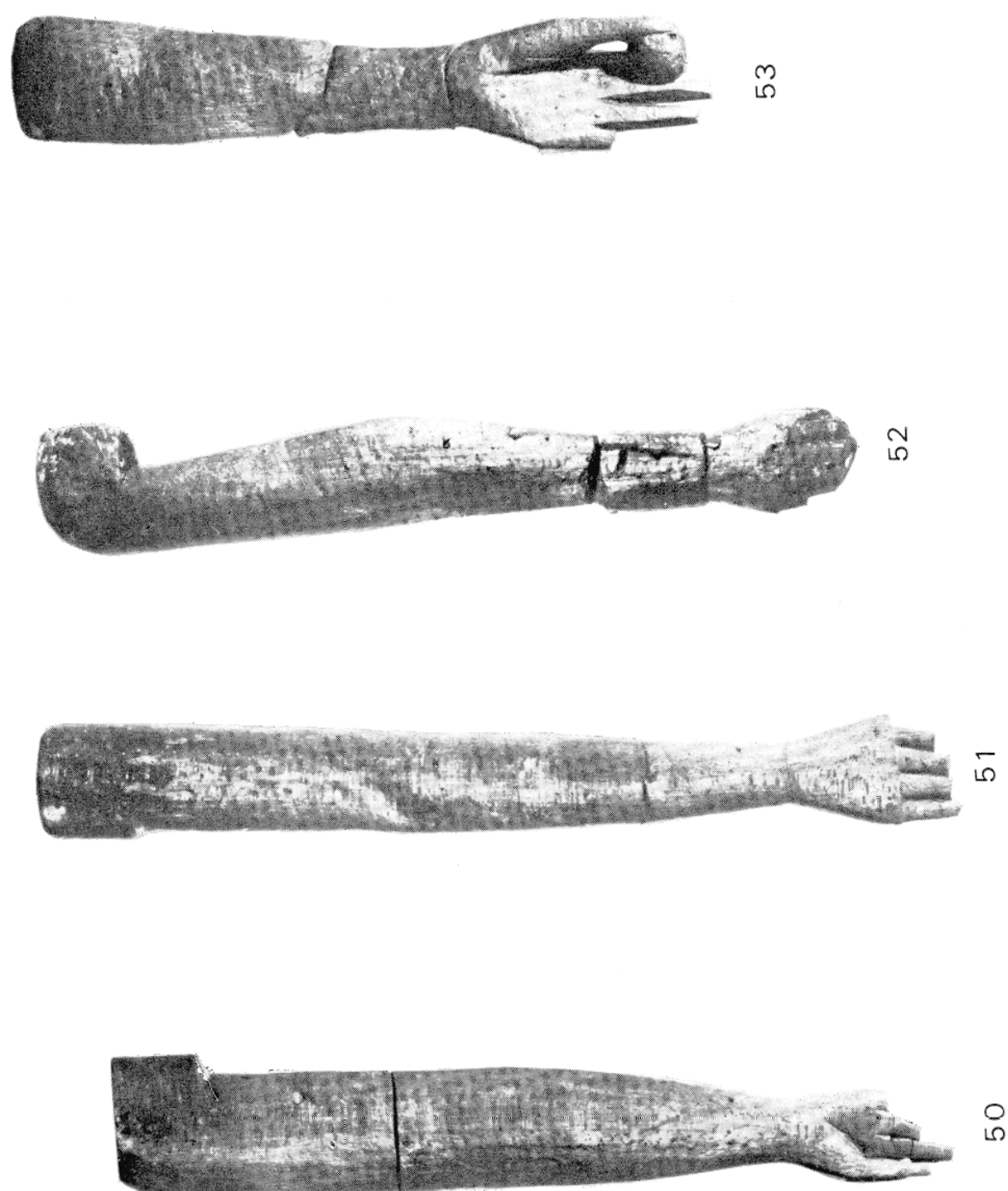

온
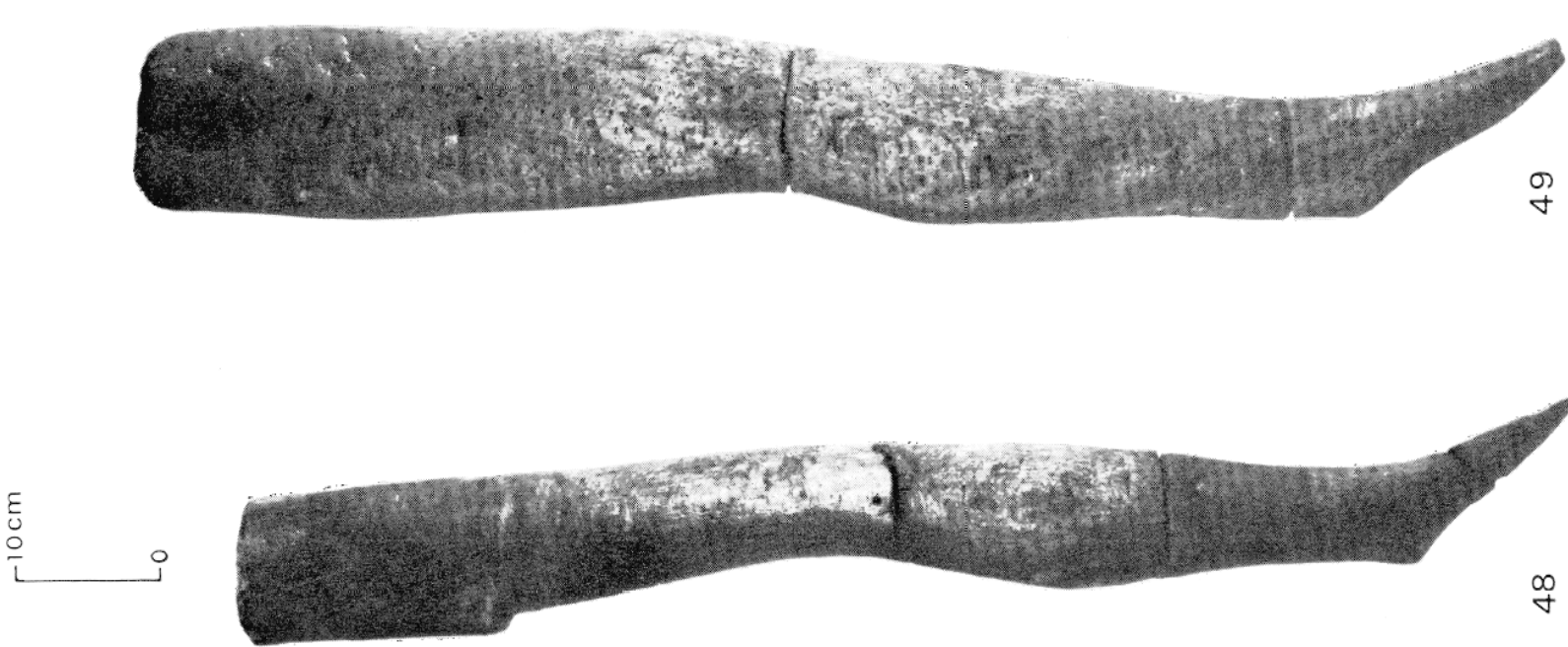
Il existe donc dans la série des têtes et bustes, comme pour les autres types d'ex-voto précédemment décrits, des analogies nombreuses entre certains exemplaires provenant des deux sites, aussi bien parmi les très belles têtes que dans le groupe de celles "de tradition celte ", et plus encore peut-être entre les naïves et frustes représentations de têtes superposées. La multitude de bustes en bois de hêtre au visage rond et plat avec des traits stéréotypés et une coiffure stylisée en godrons reste cependant très caractéristiques de Chamalières, malgré les similitudes signalées plus haut avec un unique buste féminin en bois des Sources de la Seine, et quelques points communs avec certains ex-voto de pierre du même site : forme du buste coupé au niveau des épaules, visage rond au menton lourd, stylisation des yeux et de la coiffure ${ }^{53}$.

Les ressemblances entre les deux dépôts, lorsqu'on les souligne, sont étonnantes. Toutes les catégories d'ex-voto se retrouvent, en proportions différentes certes, et quelquefois avec des particularités propres, mais sans exception sur les deux sites. Même les branches, les petits objets mobiliers, et les plaquettes, n'échappent pas à cette règle, ce qui prouve la communauté d'esprit des pèlerins fréquentant les deux sanctuaires. Quant aux représentations anatomiques d'organes internes et aux têtes superposées, on les dirait interchangeables, peut-être parce que ce sont des sculptures uniquement votives, sans rapport possible avec la grande statuaire, et qu'elles restent très liées au matériau, les premières s'accommodant bien du schéma de la planche et les dernières s'inscrivant naturellement dans la forme cylindrique de la branche. Mais des sculptures aussi caractéristiques et propres à ces deux sites impliquent aussi indiscutablement un échange de modèles ou une communication quelconque entre les artisans fournissant la clientèle des deux sanctuaires. Une pareille communauté de thème pour chaque type d'ex-voto et une telle similitude entre certaines réalisation ne peuvent être fortuites. La sculpture votive obéissait assurément à des règles spécifiques bien définies.

Il existe malgré tout des différence s importantes entre les ex-voto des deux sites qui nous intéressent. Tous ceux des Sources de la Seine sont en chêne, et presque tous sculptés en ronde-bosse, d'une qualité assez régulière, et sans "style " particulier, à quelques exceptions près; ils relèvent d'une technique simple et ont vraisemblablement peu subi d'influences extérieures ${ }^{54}$. A Chamalières au contraire, le matériau lui-même est plus diversifié $(80 \%$ des ex-voto sont en hêtre, $10 \%$ en chêne, et $10 \%$ dans des branches d'essences diverses) et le style et la qualité sont moins uniformes. Les branches ont servi à tailler des représentations très frustes (fig. 2, 6 et 8; fig. 5, 24, par exemple), plus rudes encore qu'en Bourgogne; le chêne a été employé exceptionnellement pour de très belles sculptures, d'excellente qualité et d'inspiration classique (fig. 11, 58 à 60), mais surtout pour des statues très proches de celles des Sources de la Seine (fig. 2, 4; fig. 3, 14; fig. 6, 31 et 35; fig. 7, 41) qui sont en chêne elles aussi; le hêtre, lui, a constitué le matériau privilégié des ex-voto auvergnats qui, sculptés en bas-relief, forment une collection originale.

Les ex-voto de hêtre de Chamalières ont un aspect très homogène et présentent les caractères d'une fabrication de série : un style identique imitant vraisemblablement un modèle, une technique assurée, et une grande qualité d'exécution, avec une finition, qui n'existent pas aux Sources de la Seine La confection en série paraît avoir trouvé son achèvement dans la réalisation des ex-voto de jambes (fig. 9) dont la stylisation est un modèle de réussite résultant d'une adaptation parfaite à la forme du bois débité en planches à section triangulaire : l'arête figure l'os du tibia et la partie arrondie sert à modeler le galbe du mollet, tandis que le pied est représenté en extension selon un dessin qui permet de l'inclure sans problème dans une planche de largeur relativement faible (fig. 9, 48 et 49). De même 
pour les bras, la partie mince du bois correspond à leur ligne externe ct à l'auriculaire, tandis que dans la partie épaisse est sculpté le pouce en opposition, sous l'index (fig. 9, 50 à 53), avec parfois l'offrande en relief (53). Dans les deux cas, l'économie de matériau est remarquable mais la qualité de la réalisation n'en souffre pas.

Ces remarques de fabrication s'appliquent aussi aux séries de stèles de pèlerins et de bustes à coiffure à godrons (fig. 10 ct 11). Quand les statues sont sculptées dans des planches d'épaisseur constante, elles sont symétriques, avec un aspect figé (fig. 5, 28; fig. 10, 55); mais quand, comme pour les membres, la section de la planche est dissymétrique, la partic épaisse a été généralement utilisée pour sculpter l'offrande, le bras, ou le pli du vêtement des personnages (fig. 5, 29; fig. 10, 54), tandis que les bustes tirent de cette dissymétric un aspect moins rigide, presque en mouvement (fig. 11, 61).

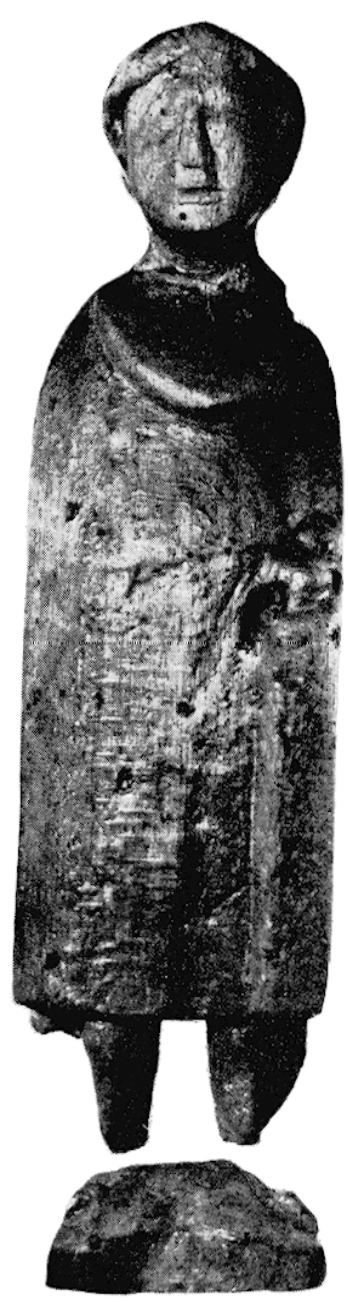

54

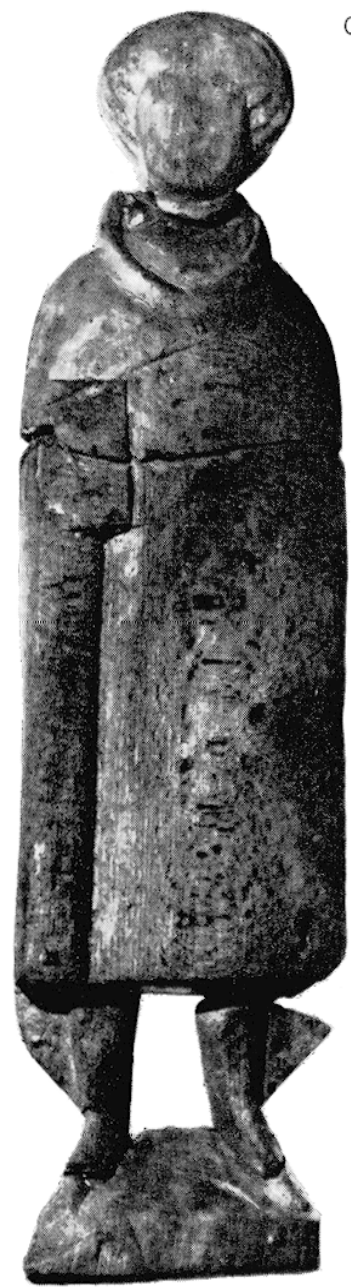

55

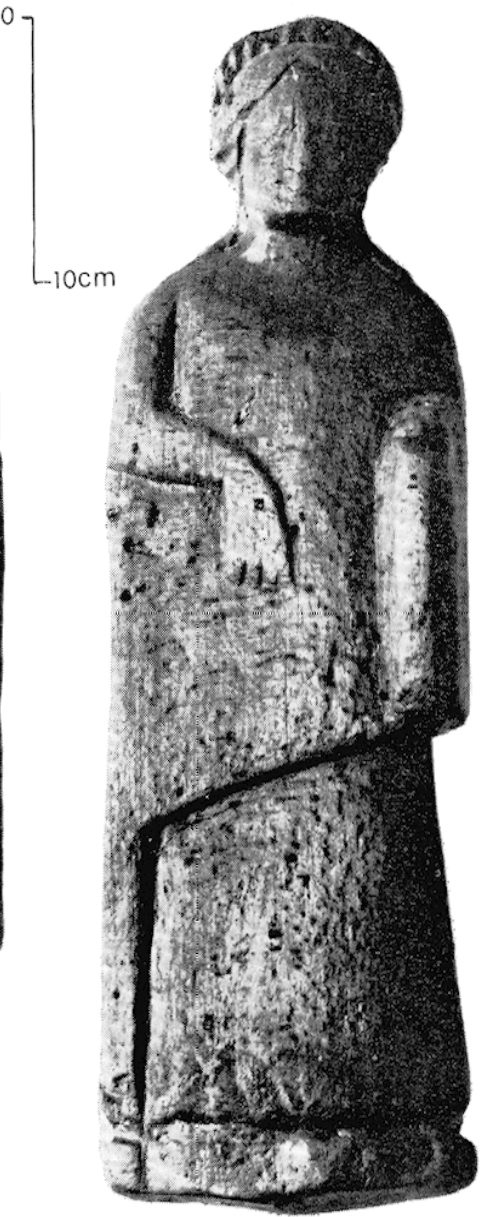

56

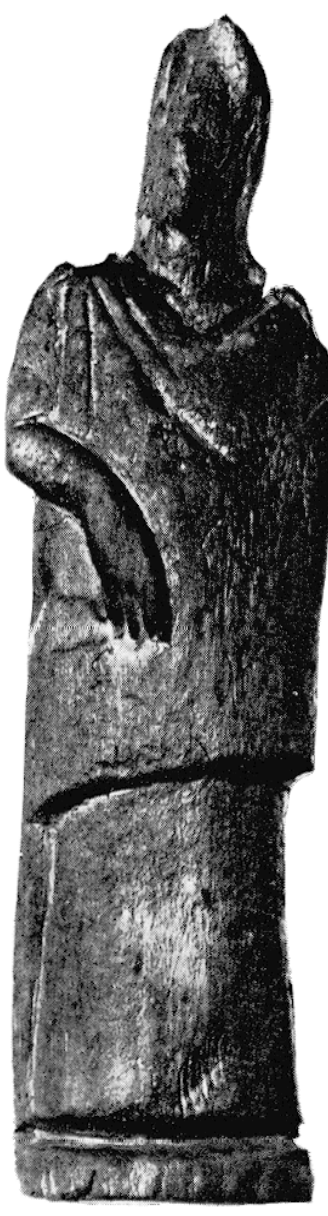

57

10 Stèles de pèlerins du "style de Chamalières ": 54, Chamalières. Personnage masculin. Haut. : 0,49 m ; 55, Chamalières. Personnage masculin. Haut. : $0,49 \mathrm{~m} ; \mathbf{5 6}$, Chamalières. Personnage féminin. Haut. : $0,46 \mathrm{~m} ; \mathbf{5}$, Chamalières. Personnage féminin. Haut. : $0,45 \mathrm{~m}$ (échelle commune). 
Ainsi s'explique, par l'emploi de planches, le peu de relief constaté sur la plupart des ex-voto de Chamalières par opposition à ceux des Sources de la Seine. Très exceptionnellement, l'arête d'un quart de rondin a été utilisée sur le devant de la sculpture pour le relief du nez et la partie épaisse à l'arrière pour la nuque et le dos, de sorte que le buste (fig. 11,62) ou le pèlerin entier ${ }^{55}$ sont alors presque en ronde bosse. L'utilisation judicieuse du mode de débitage du bois n'est donc pas douteuse, et il s'y ajoute encore, dans le cas des coiffures, l'exploitation dans un but décoratif d'une autre contrainte technique, la trace de la gouge dont le sillon figure le relief stylisé des mèches de cheveux.

Une pareille maîtrise de la lechnique, adaptée au matériau, et stéréotypée, dans un souci de rendement et d'efficacité, est incontestablement la preuve de l'existence d'un atelier spécialisé et organisé, qui avait aussi nécessairement ses modèles. S'il est difficile de déterminer l'origine de ces derniers dans le cas des membres, on peut vraisemblablement la situer en Italie pour les représentations de bustes, tant ils ressemblent parfois aux ex-voto de terre cuite de ce pays, par leurs dimensions, la forme même du buste court avec un revers plat, le schéma rond du visage au menton haut et fort, la coiffure à mèches rayonnantes et parfois l'existence d'un voile ${ }^{56}$. L'aspect de série provenant, on l'a vu, d'une volonté de rendement, à Chamalières ne faisait aussi qu'imiter celui qui résultait de l'utilisation de la technique du moulage en Italie. Et effectivement, lorsqu'ils étaient peints les uns et les autres, les ex-voto de bois devaient ressembler étonnamment à ceux de terre cuite, malgré les traits un peu plus marqués de ceux d'Italie.

Cette influence particulièrement nette pour les bustes, l'est moins pour les statuettes de personnages en pied qui, en Italie, sont plus classiques et réalistes qu'à Chamalières. S'il n'était en effet ni difficile ni long de reproduire par moulage les ex-voto en argile de personnages aux traits détaillés et au vêtement d'un drapé élaboré, les pèlerins en bois qui étaient sculptés un à un ne pouvaient l'être avec ce luxe de détail pour des raisons de rendement évidentes alors que la série des bustes devait nécessiter proportionnellement moins de travail pour un résultat plus approchant.

Les Gallo-romains ont donc adapté à la sculpture de bois, qui était leur moyen d'expression traditionnel, certains critères stylistiques de la sculpture votive italique, et, à un moindre degré, sa rapidité d'exécution pour les bustes, en se réservant une interprétation plus accordée aux contraintes du travail du bois pour les figurations de personnages entiers et même de membres. Ce style, résultant d'une parfaite adaptation au matériau jointe à une volonté stylistique, et à une exigence de qualité, explique notamment l'aspect très cocasse des moitiés inférieures de corps caractéristiques du site de Chamalières, où le bassin a été considéré comme une véritable sculpture en pied, correctement proportionnée, bien finie, et reposant sur un socle (fig. $3, \mathbf{1 6}$ ).

Les ex-voto italiques n'étaient pas inconnus des artisans bourguignons non plus, à preuve le buste féminin à coiffure à godrons et certains bustes en pierre des Sources de la Seine qui, on l'a vu, s'apparentent à ceux de Chamalières. Sur les deux sites, ce style a été appliqué principalement à un matériau spécifique, le calcaire en Bourgogne, et le hêtre en Auvergne, un matériau local abondant et facile à travailler dans les deux $\operatorname{cas}^{57}$. Mais, alors qu'il est resté limité à quelques exemplaires aux Sources de la Seine, il s'est développé avec ampleur à Chamalières, où il s'est imposé par rapport aux autres styles. La cause de ce phénomène n'est pas facile à cerner, mais il ne paraît pas absurde de supposer que c'est pour répondre à une demande massive, beaucoup plus importante qu'aux Sources de la Seine, que l'atelier s'est spécialisé dans un type de production au détriment des autres, ou qu'un atelier a monopolisé le marché aux dépens de ses concurrents.

55 Par exemple Musée Bargoin no 1.

56 Voir par exemple Enea nel Lazio, Rome, 1981 : hauteur des bustes : 20 à $30 \mathrm{~cm}$; revers plat p. 265 ; forme du buste : A 29-A 30 , p. 29 ; D 272 , p. 268 ; D 268 ; p. 252 ; D 246,247 , 248 , p. 258 ; forme ronde du visage : A 29-30, p. 29 ; A 33, p. 30 ; A 7 , p. 16 ; menton haut et fort : A 34 , p. 30 ; I) 258 , p. 262 ; coiffure stylisée en mèches raides et symétriques : A 7, p. 43 ; D 268, 269, 270, 271, 272, p. 268; voile: A 29, A 30, A 33, p. 30.

57 Il est difficile tant que les ex-voto de pierre des Sources de la Scine ne seront pas datés avec certitude d'envisager des raisons chronologiques à cet état de faits. 

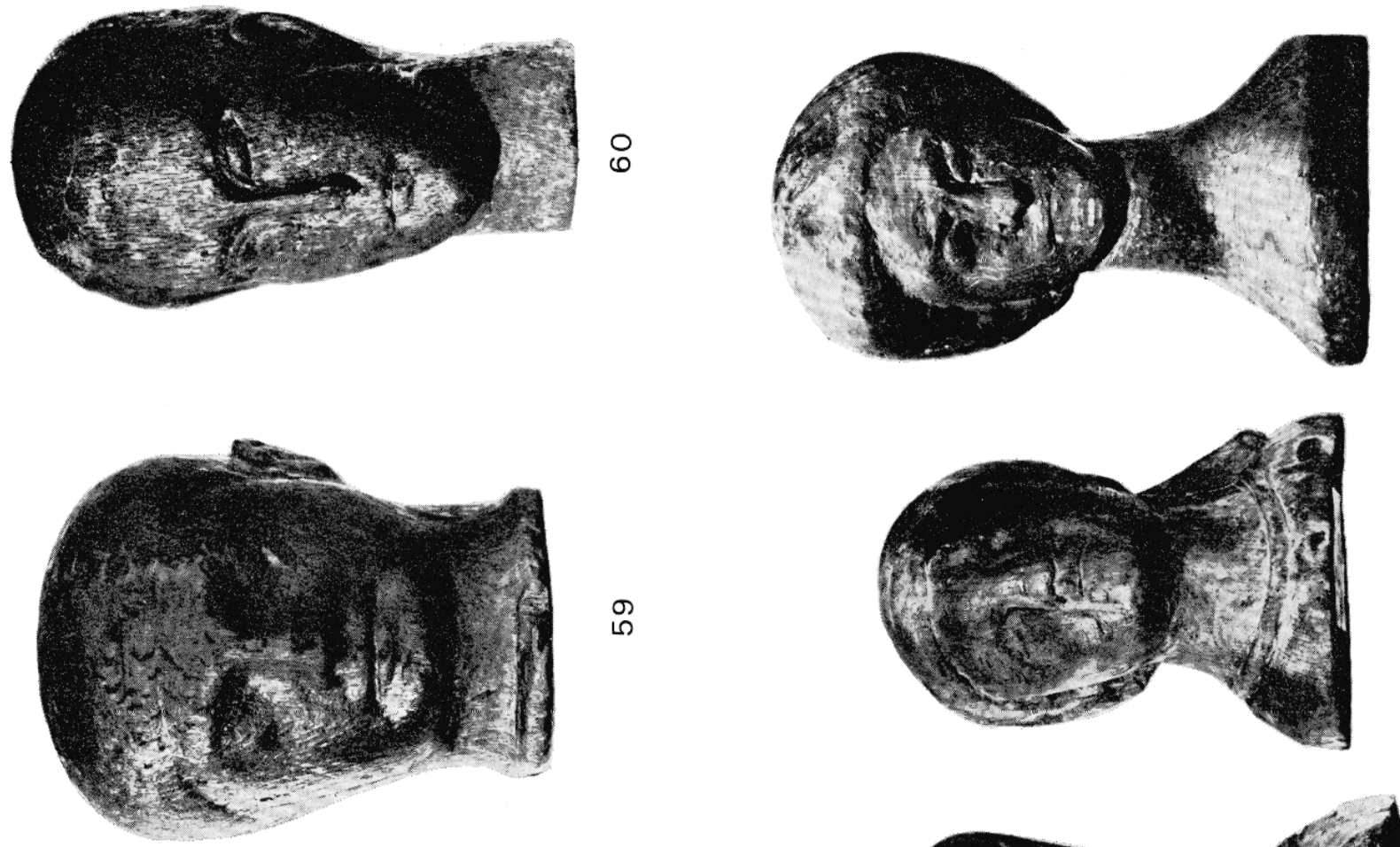

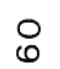

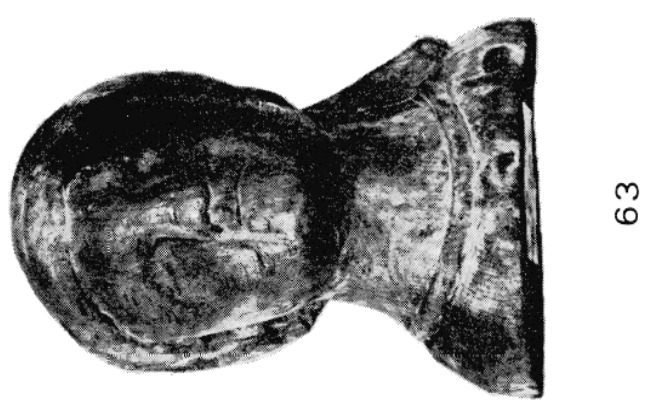

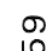
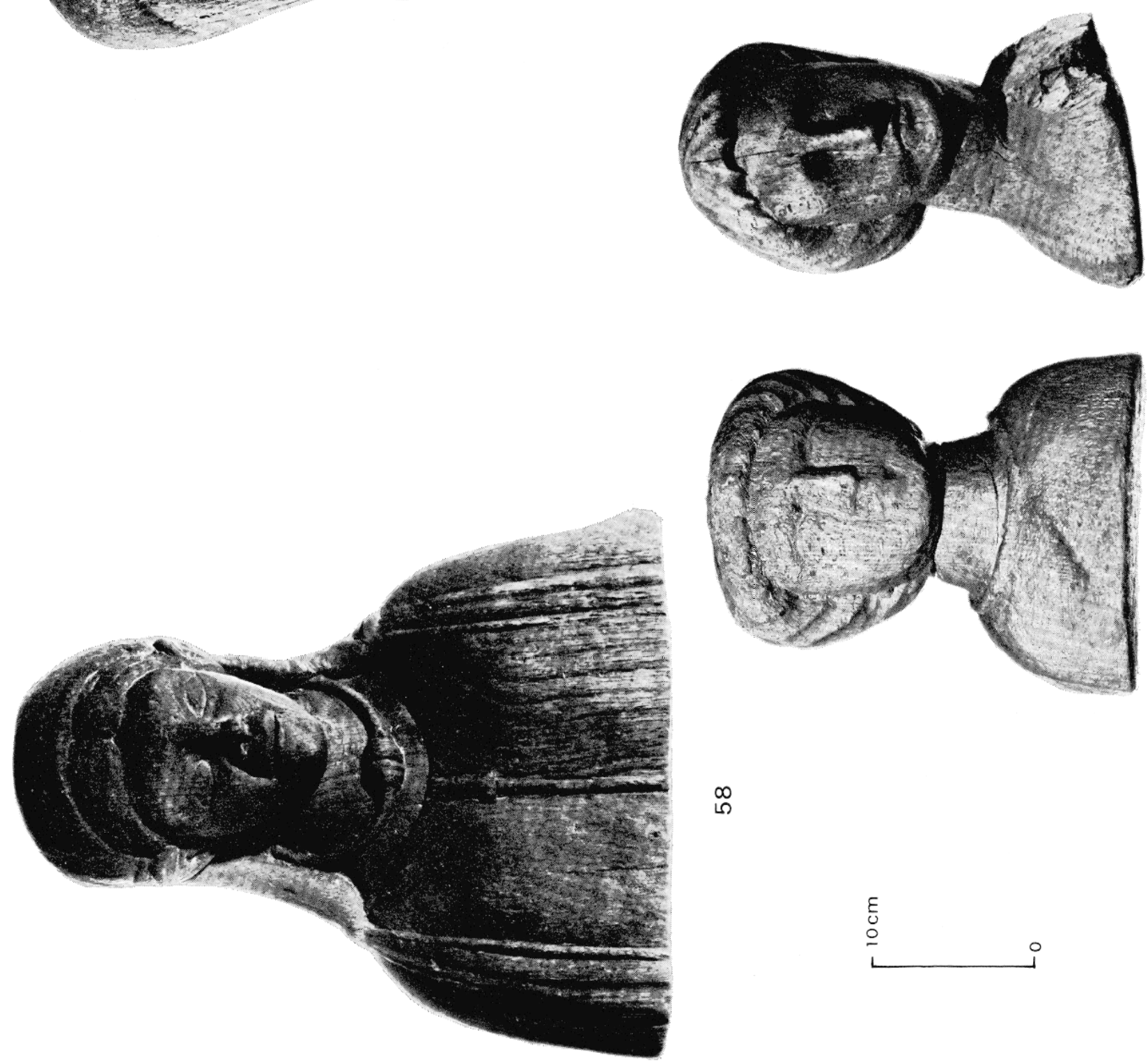

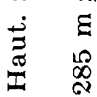

- $0 \dot{\bar{\Xi}}$

苛

I.

号

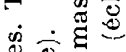

边

ฮี है

要

คิ

ह

용

$\circ 0$.

$\because 0$.

ช

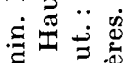

ยี

ษ 콣

品政

है

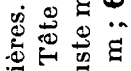

สำ

象象

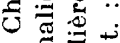

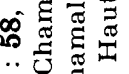

б

政

世

范

है

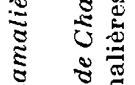

ड ธั ธี

$\approx$ के

ڤ

₹

2
$\approx$
$\approx$ 


\begin{tabular}{|c|c|c|c|c|}
\hline \multicolumn{5}{|c|}{ NOMBRE D'EX-VOTO TYPE PAR TYPE SUR CHAQUE SITE } \\
\hline \multirow{2}{*}{ TyPES } & \multirow{2}{*}{$\begin{array}{c}\text { Chamalières (sur les } \\
1550 \text { ex-voto identifiés } \\
\text { avec certitude) }\end{array}$} & \multicolumn{3}{|c|}{ SOURCES DE LA SEINE } \\
\hline & & Bois & Pierre & TOTAL \\
\hline JAMBES et PIEDs.............. & 670 & 45 & 100 & 145 \\
\hline 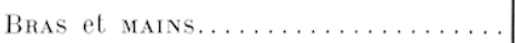 & 300 & 11 & 54 & 65 \\
\hline Personnages entiers............. & 245 & 50 & 55 & 105 \\
\hline Tètes et Bustes.................. & 145 & 63 & 125 & 188 \\
\hline TÊTES GROUPÉES................. & 3 (superposées) & $\begin{array}{c}17 \\
\text { (superposées ou } \\
\text { opposées) }\end{array}$ & $\begin{array}{c}2 \\
(\text { juxtaposées) }\end{array}$ & 19 \\
\hline 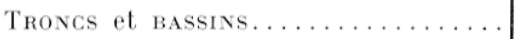 & 40 & 13 & 39 & 52 \\
\hline Planches anatomigues............. & 15 & 53 & - & 53 \\
\hline ANimaUx ou pattes ou protomes...... & 12 & 25 & 5 & 30 \\
\hline 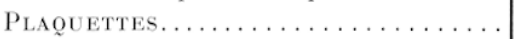 & 60 & "nombreuses" & - & "nombreuses" \\
\hline ObJets mobiliers............... & 45 & 9 & - & 9 \\
\hline Branches $\ldots \ldots \ldots \ldots \ldots \ldots \ldots$ & nombreuses & nombreuses & - & nombreuses \\
\hline
\end{tabular}

N.B. Aucun des chiffres concernant Chamalières n'est absolument définitif. Ils ont, pour les plus importants, été arrondis.

La comparaison entre les ex-voto provenant des deux sites met donc en évidence des courants commerciaux et religieux entre les deux sanctuaires, et une communauté d'esprit entre leurs clientèles, toutes deux indigènes - les inscriptions en langue celtique l'attestent ${ }^{58}-$, puisque l'on trouve sur l'un et l'autre lieux, non seulement les mêmes séries d'ex-voto, mais des sculptures de styles comparables. Cependant l'influence italique, qui existe en Bourgogne, a été répercutée avec beaucoup plus d'ampleur à Chamalières, soit pour des raisons uniquement numériques, soit par le fait de la prédominance d'un atelier. La découverte des Sources de la Seine a révélé une sculpture originale pratiquement inconnue jusqu'alors, celle de Chamalières a montré son abondance et son achèvement en un site donné.

\section{Anne-Marie Romeuf}

N.B. Les photographies des ex-voto des sources de la Seine nous ont aimablement été communiquées par Simone Deyts que nous remercions pour sa libéralité. Celles des bois de Chamalières sont l'œuvre de MM. Dubois et Levasseur, photographes à la Faculté des Lettres de Clermont-Ferrand.

58 Aux Sources de la Seine : M. Lejeune et R. Marichal, Stèle inscrile des Sources de la Seine, dans Revue Archéologique de l'Est, t. LVIII, no 1-2, 1956, p. 71-82. - A Chamalières, M. LEJEUnE et R. MARICHAL, Textes gaulois et gallo-romains en cursive latine, dans Eludes Celtiques, XV, 1, 1976-77, p. 156-158 et pl. XIV. - L. FleURIOT, Le vocabulaire de l'inscription gauloise de Chamalières, dans Etudes Celtiques, $\mathrm{XV}, 1,1976-77$, p. 173-190; ID., Nole additionnelle sur l'inscription de Chamalières, dans Études Celtiques, XVI, 1979 , p. 135-140. - P.-Y. LAMBERT, La tablette gauloise de Chamalières, dans Etudes Celtiques, XVI, 1979, p. 141-169. 